\title{
Regularity of the transfer map for cohomologous cocycles
}

\author{
VIOREL NIŢICĂ $\dagger$ and ANDREI TÖRÖK $\ddagger$ \\ Institute of Mathematics of the Romanian Academy, PO Box 1-764, \\ RO-70700 Bucharest, Romania
}

(Received 17 August 1996 and accepted in revised form 10 September 1997)

\begin{abstract}
In this paper we obtain results about the regularity of the transfer map between two cocycles over an Anosov system, with values in either a diffeomorphism or a Lie group. We also explain how certain examples of de la Llave show that our results are essentially optimal.
\end{abstract}

\section{Introduction}

Let $G$ be a group acting on a compact Riemannian manifold without boundary $M$ by $\alpha: G \times M \rightarrow M,(g, x) \mapsto \alpha_{g}(x) \equiv g x$. Let $\Gamma$ be some topological group. A cocycle $\beta$ over the action $\alpha$ is a continuous function $\beta: G \times M \rightarrow \Gamma$ such that

$$
\beta\left(g_{1} g_{2}, x\right)=\beta\left(g_{1}, g_{2} x\right) \beta\left(g_{2}, x\right),
$$

for all $g_{1}, g_{2} \in G, x \in M$.

A geometric interpretation of a cocycle is the following. Consider the trivial principal $\Gamma$-bundle $E=M \times \Gamma$ over $M$. Then the cocycle $\beta$ described above corresponds to a lift of the action $\alpha$ to an action $\tilde{\alpha}: G \times E \rightarrow E$ by principal bundle maps. Namely, $g \in G$ induces the map $\tilde{\alpha}_{g}: E \rightarrow E$ given by $(x, h) \mapsto\left(\alpha_{g}(x), \beta(g, x) h\right)$. The cocycle equation (1.1) is equivalent to the fact that $\tilde{\alpha}$ is an action, i.e. $\tilde{\alpha}_{g_{1}} \tilde{\alpha}_{g_{2}}=\tilde{\alpha}_{g_{1} g_{2}}$.

If $\Gamma=\operatorname{Aut}(F)$ for some space $F$, then a cocycle $\beta: G \times M \rightarrow \Gamma$ also corresponds to a lift of $\alpha$ to an action by bundle maps on the trivial bundle $M \times F$. In this case $g \in G$ acts by $(x, \xi) \mapsto\left(\alpha_{g}(x), \beta(g, x)(\xi)\right)$. Here 'Aut(.)' has the meaning appropriate for the structure of $F$. It can be GL(.) for $F$ a linear space, or Diff(.) for $F$ a manifold.

The natural equivalence relation for cocycles is the cohomology. Two cocycles $\beta_{1}$ and $\beta_{2}$ are called cohomologous if there exists a continuous map $P: M \rightarrow \Gamma$ such that

$$
\beta_{1}(g, x)=P(g x) \beta_{2}(g, x) P(x)^{-1},
$$

$\dagger$ Current address: Department of Mathematics, Pennsylvania State University, University Park, PA 16802, USA. (e-mail: nitica@math.psu.edu)

$\ddagger$ Current address: Department of Mathematics, Princeton University, Princeton, NJ 08544-1000, USA. (e-mail: torok@math.princeton.edu) 
for all $g \in G, x \in M$.

We call $P$ a transfer map between $\beta_{1}$ and $\beta_{2}$. We will refer to (1.2) as the cohomology equation.

In terms of the geometric interpretations given above, two cocycles are cohomologous if the two induced lifts of the action are conjugated through a (principal) bundle map:

$$
\tilde{\alpha}_{1}(g)=\mathbf{P} \circ \tilde{\alpha}_{2}(g) \circ \mathbf{P}^{-1}, \quad g \in G,
$$

where $\mathbf{P}(x, y):=(x, P(x) y)$ and $\tilde{\alpha}_{1}, \tilde{\alpha}_{2}$ are the lifts corresponding to $\beta_{1}, \beta_{2}$.

A cocycle $\beta: G \times M \rightarrow \Gamma$ is called cohomologically trivial if the equation

$$
\beta(g, x)=P(g x) P(x)^{-1}
$$

has a continuous solution $P: M \rightarrow \Gamma$. We will refer to this equation as the Livsic cohomology equation.

The following question turns out to be quite important for many rigidity results: what can be said about the regularity of the solution $P$ of the Livsic cohomology equation, provided that $\beta$ and the action $\alpha$ are $C^{K}(K=1,2, \ldots, \omega)$ ?

Results in this direction were first obtained by Livsic, for real valued cocycles over Anosov $\mathbb{Z}$-actions. He showed that if the cocycle $\beta$ is $C^{1}$, then the trivialization $P$ is also $C^{1}[\mathbf{L 1}]$. For some linear actions on a torus he also showed that if the cocycle is $C^{\infty}$, respectively $C^{\omega}$, then so is the solution [L2]; this was obtained by studying the decay of the Fourier coefficients.

Later, Guillemin and Kazhdan [GK1, GK2] showed $C^{\infty}$-regularity of the solutions in the case of geodesic flows on negatively curved surfaces. Collet et al [CEG] proved a $C^{\omega}$ version for geodesic flows of constant negative curvature.

The final conclusion for the $C^{\infty}$ case appears in the paper by de la Llave et al [LMM]. One of the technical results involved is that if a function is smooth along two transverse foliations which are absolutely continuous and whose Jacobians have some regularity properties, then it is smooth globally. This was proved in [LMM] using properties of elliptic operators. Later, a more general result was proved by Journé $[\mathbf{J}]$ (see Theorem 5.1 in the sequel), relying mainly on Taylor expansions and the estimate of the error. Another approach is presented in Hurder and Katok [HuK], based on an unpublished idea of C. Toll. Here the decay of the Fourier coefficients is used to characterize smoothness. The method can be applied for spanning families of foliations which have the same property as those used in $[\mathbf{L M M}]$. Note that foliations arising from Anosov diffeomorphisms have this property. Using the approach in $[\mathbf{H u K}]$, de la Llave proved the analytic case in [Lla1].

In [NT2] the same question was considered for cocycles taking values in Lie and diffeomorphism groups.

Notice that for $\Gamma$ an abelian group, the cohomology equation (1.2) can be restated in terms of a cohomologically trivial cocycle. However, for a general $\Gamma$, one has to address the following question: if two $C^{K}$-cocycles are cohomologous through a continuous transfer map $P$, what can be said about the regularity of $P$ ?

The goal of this paper is to answer this question in some instances for $\Gamma$ a Lie group or a group of diffeomorphisms. It is organized as follows. In $\S 2$ we state the main results; 
in $\S 3$ some facts about (partially) hyperbolic diffeomorphisms are given, followed in $\S 4$ by a counterexample due to de la Llave and a variant of it which show that results of this type cannot be improved; the proofs are given in $\S \S 5$ and 6. The Remark in $\S 5$ discusses the difference between our approach and the one used for the previous results. We finish with an Appendix.

Notation. In the sequel $\mathcal{A}$ stands for either $\mathbb{Z}$ or $\mathbb{R}$. In order to be able to refer to both cases simultaneously, the elements of an $\mathcal{A}$-action are denoted by $s \in \mathcal{A} \mapsto T^{s}$. Therefore, $T^{1}$ is either the time-one map of a flow or the diffeomorphism generating a $\mathbb{Z}$-action.

\section{The main results}

Let $M$ and $N$ be compact manifolds, and consider an Anosov action $T: \mathcal{A} \times M \rightarrow M$ (i.e. an Anosov diffeomorphism for $\mathcal{A}=\mathbb{Z}$ and an Anosov flow for $\mathcal{A}=\mathbb{R}$; see $\S 3$ for the definitions). Let $\beta, \tilde{\beta}: \mathcal{A} \times M \rightarrow \operatorname{Diff}^{K}(N)$ be two $C^{K}$ cocycles which are cohomologous through the transfer map $P: M \rightarrow \operatorname{Diff}^{K}(N)$, i.e.

$$
\beta(g, x)=P(g x) \tilde{\beta}(g, x) P(x)^{-1} .
$$

For $G$ a finite-dimensional Lie group one can also consider two $C^{K}$ cocycles $\beta, \tilde{\beta}$ : $\mathcal{A} \times M \rightarrow G$ which are cohomologous through a transfer map $P: M \rightarrow G$.

We will discuss regularity results for the solution $P$ of equation (2.1), both for Diffand Lie group valued cocycles. In some cases the results generalize those of [NT2].

To obtain the regularity of the transfer map, we have to require that it is already Hölder of some order. That this condition is essential follows from $\S 4$. The examples presented there also show that Theorems 2.2 and 2.4 are in a sense optimal.

We first define continuous or differentiable Diff-valued functions.

Definition 2.1. Assume that $K=1,2, \ldots, \omega$ and $\alpha \in[0,1)$. Let $f: \mathcal{A} \times M \rightarrow \operatorname{Diff}^{K}(X)$ be a function. One can identify $f$ with a function $\bar{f}: \mathcal{A} \times M \times X \rightarrow X$.

We say that $f$ is continuous if it is continuous into the $C^{K}$-topology on $\operatorname{Diff}^{K}(X)$. We say that $f$ is $C^{K+\alpha}$ if $\bar{f}$ is $C^{K+\alpha}$.

THEOREM 2.2. Let $M$ and $N$ be compact Riemannian manifolds, and let $\beta, \tilde{\beta}: \mathcal{A} \times M \rightarrow$ $\operatorname{Diff}^{K}(N)$ be two $C^{K}$ cocycles over the $C^{K}$ Anosov action $T: \mathcal{A} \times M \rightarrow M$, where $K=1,2, \ldots, \omega$. Assume that $\beta$ and $\tilde{\beta}$ are cohomologous through a continuous transfer map $P: M \rightarrow \operatorname{Diff}^{K}(N)$. Denote

$$
\begin{aligned}
& \lambda_{-}=\lim _{n \rightarrow \infty}\left\|\left.D T^{n}\right|_{E^{s}}\right\|^{1 / n}, \\
& \lambda_{+}=\lim _{n \rightarrow \infty}\left\|\left.D T^{-n}\right|_{E^{u}}\right\|^{-1 / n}, \\
& \mu_{+}=\lim _{n \rightarrow \infty} \sup _{x \in M}\left\|D_{N} \beta(n, x)\right\|^{1 / n}, \\
& \mu_{-}=\lim _{n \rightarrow \infty} \inf _{x \in M}\left\|D_{N} \beta(n, x)^{-1}\right\|^{-1 / n},
\end{aligned}
$$


where $T M=E^{s} \oplus E^{0} \oplus E^{u}$ is the $\mathcal{A}$-invariant splitting of $T M$. Note that $\mu_{-} \leq \mu_{+}$. Assume that $\lambda_{-}<\mu_{-} \leq \mu_{+}<\lambda_{+}$, and set

$$
\alpha_{0}=\max \left\{\frac{\ln \mu_{+}}{\ln \lambda_{+}}, \frac{\ln \mu_{-}}{\ln \lambda_{-}}\right\} .
$$

If $\alpha_{0}=0$ (possible only if $\mu_{-}=\mu_{+}=1$ ) and

$$
\begin{aligned}
& \limsup _{n \rightarrow \infty} \sup _{x \in M}\left\|D_{N} \beta(n, x)\right\|<\infty, \\
& \limsup _{n \rightarrow \infty} \sup _{x \in M}\left\|D_{N} \beta(n, x)^{-1}\right\|<\infty,
\end{aligned}
$$

then $P: M \rightarrow \operatorname{Diff}^{K}(N)$ is $C^{K-\varepsilon}$ for any small $\varepsilon>0$.

In general, if $P: M \times N \rightarrow N$ is $\alpha$-Hölder for some $\alpha>\alpha_{0}$, then $P: M \rightarrow \operatorname{Diff}^{K}(N)$ is $C^{K-\varepsilon}$ for any small $\varepsilon>0$. (For $K \in\{1, \infty, \omega\}, K-\varepsilon:=K$.)

Remarks. 0. By the spectral radius theorem, the limits defining $\lambda_{ \pm}$and $\mu_{ \pm}$exist and equal the bounds of the spectra of $D T^{1}$ and $D \tilde{T}_{\beta}^{1}$ on the complexification of $T M$, respectively of the kernel of the natural projection $T(M \times N) \rightarrow T M$ (here $\tilde{T}_{\beta}^{t}$ stands for the lift of $T^{t}$ to $M \times N$ via $\beta$ ). See also $\S 3$.

1. The relation $\lambda_{-}<\mu_{-} \leq \mu_{+}<\lambda_{+}$holds for cocycles that are close enough to $\operatorname{Id}_{N}$. Indeed, $\mu_{+} \leq \sup _{x \in M}\left\|D_{N} \beta(1, x)\right\|$ and $\mu_{-} \geq \inf _{x \in M}\left\|D_{N} \beta(1, x)^{-1}\right\|^{-1}$.

2. By (2.1) and the boundedness of $D_{N} P_{x}^{ \pm 1}$, the quantities $\mu_{ \pm}$are the same for $\beta$ and $\tilde{\beta}$. The same is true about condition (2.2).

3. One can get a statement similar to Theorem 2.2 for cocycles taking values in a compact Lie group $\Gamma$, because letting $\Gamma$ act on itself by left translations gives an inclusion $\Gamma \hookrightarrow \operatorname{Diff}^{\omega}(\Gamma)$ of $\Gamma$ into the group of diffeomorphisms of a compact manifold. Note that in this case $\alpha_{0}=0$.

By taking $\beta \equiv \operatorname{Id}_{N}$ one obtains the following strengthening of [NT2, Theorem 2].

Corollary 2.3. Let $K, N$ and $T: \mathcal{A} \times M \rightarrow M$ be as in Theorem 2.2. Assume that $\tilde{\beta}: \mathcal{A} \times M \rightarrow \operatorname{Diff}^{K}(N)$ is a $C^{K}$-cocycle which is cohomologically trivialized by the continuous transfer map $P: M \rightarrow \operatorname{Diff}^{K}(N)$. Then $P$ is $C^{K-\varepsilon}$ for any small $\varepsilon>0$.

As an application of Theorem 2.2, we obtain the following result for Lie groups. The conditions are more restrictive than in Theorem 2.2 (see Remark 3 after Theorem 2.4).

THEOREM 2.4. Assume that $G$ is a (closed subgroup in a) finite-dimensional Lie group that admits a cocompact lattice. Let $K, T: \mathcal{A} \times M \rightarrow M$ and $\lambda_{ \pm}$be as in Theorem 2.2 and let $\beta, \tilde{\beta}$ be $C^{K}$-cocycles taking values in $G$ which are cohomologous through a continuous transfer map $P: M \rightarrow G$. Denote

$$
\begin{aligned}
& \mu_{+}=\lim _{n \rightarrow \infty} \sup _{x \in M}\left\|\operatorname{Ad}_{\beta(n, x)}\right\|^{1 / n}, \\
& \mu_{-}=\lim _{n \rightarrow \infty} \inf _{x \in M}\left\|\operatorname{Ad}_{\beta(n, x)^{-1}}\right\|^{-1 / n},
\end{aligned}
$$

where $\mathfrak{g}$ is the Lie algebra of $G, \operatorname{Ad}: G \rightarrow \operatorname{Aut}(\mathfrak{g})$ with $\operatorname{Ad}_{g}$ being the differential at the identity of the inner automorphism $h \in G \mapsto \mathrm{ghg}^{-1}$, and $\|\cdot\|$ denotes the operator norm 
on Aut(g) with respect to some fixed norm on $\mathfrak{g}$. Assume that $\lambda_{-}<\mu_{-} \leq \mu_{+}<\lambda_{+}$, and set

$$
\alpha_{0}=\max \left\{\frac{\ln \mu_{+}}{\ln \lambda_{+}}, \frac{\ln \mu_{-}}{\ln \lambda_{-}}\right\} .
$$

If $\alpha_{0}=0$ (possible only if $\mu_{-}=\mu_{+}=1$ ) and

$$
\begin{aligned}
& \limsup _{n \rightarrow \infty} \sup _{x \in M}\left\|\operatorname{Ad}_{\beta(n, x)}\right\|<\infty, \\
& \limsup _{n \rightarrow \infty} \sup _{x \in M}\left\|\operatorname{Ad}_{\beta(n, x)^{-1}}\right\|<\infty,
\end{aligned}
$$

then $P: M \rightarrow G$ is $C^{K-\varepsilon}$ for any small $\varepsilon>0$.

In general, if $P: M \rightarrow G$ is $\alpha$-Hölder for some $\alpha>\alpha_{0}$, then $P$ is $C^{K-\varepsilon}$ for any small $\varepsilon>0$. (Note that since the image of $P$ is compact in $G$, the fact that $P$ is $\alpha$-Hölder is independent of the smooth metric chosen on $G$.)

Proof. Endow $G$ with a right invariant metric. Assume that $\Gamma$ is a cocompact lattice of $G$, and let $N=G / \Gamma$, with the induced metric. Since $G$ acts on $N$ by left multiplication, $P$ and the cocycles $\beta$ and $\tilde{\beta}$ can be seen as taking values in $\operatorname{Diff}^{\omega}(N)$. The values $\mu_{ \pm}$ defined for $\operatorname{Ad}_{\beta}$ coincide with the values introduced in Theorem 2.2 for the corresponding $\operatorname{Diff}(N)$-valued cocycles.

Remarks. 1. Remarks 1 and 2 after Theorem 2.2 are valid here too, applied to $\operatorname{Ad}_{\beta}$.

2. By a result of Borel $[\mathbf{B}], \operatorname{GL}(d, \mathbb{R}) \cong \mathbb{Z}_{2} \times \mathbb{R} \times \operatorname{SL}(d, \mathbb{R})$, as well as any semisimple Lie group, admits a cocompact lattice.

3. For $G$ a closed subgroup of $\operatorname{GL}(d, \mathbb{R})$ and $\beta$ a $G$-valued cocycle, consider

$$
\begin{aligned}
& \tilde{\mu}_{+}=\lim _{n \rightarrow \infty} \sup _{x \in M}\|\beta(n, x)\|^{1 / n}, \\
& \tilde{\mu}_{-}=\lim _{n \rightarrow \infty} \inf _{x \in M}\left\|\beta(n, x)^{-1}\right\|^{-1 / n},
\end{aligned}
$$

where $\|\cdot\|$ denotes the matrix norm. Then

$$
\tilde{\mu}_{-} \cdot \tilde{\mu}_{+}^{-1} \leq \mu_{-} \leq \mu_{+} \leq \tilde{\mu}_{+} \cdot \tilde{\mu}_{-}^{-1} .
$$

One obtains the following generalization of [NT2, Theorem 3].

Corollary 2.5. Let $G, K$ and $T: \mathcal{A} \times M \rightarrow M$ be as in Theorem 2.4. Assume that $\beta, \tilde{\beta}: \mathcal{A} \times M \rightarrow G$ are two $C^{K}$-cocycles which are cohomologous through a continuous transfer map $P: M \rightarrow G$. If one of the cocycles takes values in a compact subgroup of $G$, then $P$ is $C^{K-\varepsilon}$ for any small $\varepsilon>0$.

\section{Partially hyperbolic diffeomorphisms}

We first recall the definition of an Anosov diffeomorphism, respectively flow.

Let $M$ be a compact manifold and $T: \mathcal{A} \times M \rightarrow M$ a $C^{1}$-action $(\mathcal{A}=\mathbb{Z}$ corresponds to a diffeomorphism, $\mathcal{A}=\mathbb{R}$ to a flow). The action $T$ is called Anosov if there is a continuous invariant splitting of the tangent bundle $T M=E^{s} \oplus E^{0} \oplus E^{u}$ and constants $C>0, \chi_{-}<0<\chi_{+}$such that for $t \in \mathcal{A}, t \geq 0$,

$$
\begin{aligned}
\left\|D T^{t} v^{s}\right\| & \leq C e^{t \chi-}\left\|v^{s}\right\|, \quad v^{s} \in E^{s}, \\
\left\|D T^{-t} v^{u}\right\| & \leq C e^{-t \chi+}\left\|v^{u}\right\|, \quad v^{u} \in E^{u},
\end{aligned}
$$


where if $\mathcal{A}=\mathbb{Z}$ then $E^{0}=\{0\}$ and if $\mathcal{A}=\mathbb{R}$ then $E^{0}$ is spanned by the nowhere vanishing vector field which generates the flow.

The sub-bundles $E^{s}$ and $E^{u}$ are called stable, respectively unstable, distributions. These distributions are integrable. We denote by $W^{s}(x)$ and $W^{u}(x)$ the stable, respectively unstable, manifolds of the point $x \in M$. If the diffeomorphism $T^{1} \in$ $C^{K}(M)$, then the stable and unstable foliations are also $C^{K}, K=1,2, \ldots, \omega$ (see Theorem 3.1).

Note that by a $C^{K}$-foliation $(K=1,2, \ldots, \omega)$ we mean a $C^{0}$-foliation whose leaves are embedded $C^{K}$-submanifolds, and these submanifolds depend continuously in the $C^{K}$-topology of embeddings on the point through which the leaf is considered. (See Definition 6.2 for the $C^{\omega}$-topology.) By two transverse foliations we mean two $C^{1}$-foliations whose tangent spaces have trivial intersection at each point.

We now give the definition of a partially hyperbolic diffeomorphism. Our definition relies on the Mather spectrum. Note that in our application the center distribution is smooth and integrable, hence one could use the setup of Hirsch et al [HPS], where what we describe corresponds to an absolutely 1-normally hyperbolic map.

Let $M$ be a compact Riemannian manifold and $f$ a $C^{1}$-diffeomorphism of $M$. We consider the Banach space $\Gamma^{0}(M)$ of continuous vector fields on $M$, on which $f$ acts as an invertible bounded linear operator $f_{*}$. We complexify $\Gamma^{0}(M)$. The Mather spectrum $\sigma(f)$ of $f$ is the spectrum of $f_{*}$ on this complex Banach space. If the non-periodic points of $f$ are dense in $M$ then $\sigma(f)$ is a union of circles $\{|z|=a\}$. See [M].

$f$ is a partially hyperbolic diffeomorphism if there are constants $0<\lambda_{-}<\mu_{-} \leq$ $\mu_{+}<\lambda_{+}, \lambda_{-}<1<\lambda_{+}$, such that the spectrum of $f$ is contained in the union of the rings $\left\{z \in \mathbb{C}:|z| \geq \lambda_{+}\right\},\left\{z \in \mathbb{C}: \mu_{-} \leq|z| \leq \mu_{+}\right\}$and $\left\{z \in \mathbb{C}:|z| \leq \lambda_{-}\right\}$.

This is equivalent to the fact that there is an $f$-invariant continuous splitting $T M=E^{s} \oplus E^{0} \oplus E^{u}$ with the property that given any small $\delta>0$ there is a constant $C>0$ such that for any $k \geq 0$

$$
\begin{aligned}
\left\|\left.D f^{k}\right|_{E^{s}}\right\| & \leq C\left(\lambda_{-}+\delta\right)^{k}, \\
\left\|\left.D f^{-k}\right|_{E^{u}}\right\| & \leq C\left(\lambda_{+}-\delta\right)^{-k}, \\
\left\|\left.D f^{-k}\right|_{E^{0}}\right\| & \leq C\left(\mu_{-} \delta\right)^{-k}, \\
\left\|\left.D f^{k}\right|_{E^{0}}\right\| & \leq C\left(\mu_{+}+\delta\right)^{k} .
\end{aligned}
$$

As in the case of Anosov diffeomorphisms, for any fixed small $\delta>0$, one can find another smooth Riemannian metric, equivalent to the initial one, for which $C=1$ (see $[\mathbf{M}])$. Notice that, according to our definition, both an Anosov diffeomorphism and the time-one map of an Anosov flow are partially hyperbolic.

The following theorem shows that the distributions $E^{s}, E^{u}$ are integrable and characterize the leafs of the resulting foliations. It summarizes and extends some results from $[\mathbf{P}]$ and $[\mathbf{H P S}]$.

THEOREM 3.1. ([P, HPS $]$ )

(a) Assume that $M$ is a compact Riemannian manifold and $f \in \operatorname{Diff}^{K}(M)$ is a partially hyperbolic diffeomorphism $(K=1,2, \ldots, \omega)$. Then each of the distributions $E^{s}$ and $E^{u}$ is integrable. The corresponding foliations are called the stable, respectively 
unstable, foliations, and are denoted by $W^{s}$, respectively $W^{u}$. The leaves $W^{s}(x)$ and $W^{u}(x)$ are $C^{K}$, and depend continuously on the point $x \in M$ in the $C^{K}$ topology.

These foliations can be characterized as follows. Let $\lambda_{-}<\nu_{-}<\min \left\{\mu_{-}, 1\right\} \leq$ $\max \left\{\mu_{+}, 1\right\}<v_{+}<\lambda_{+}$. Then:

$$
\begin{aligned}
& y \in W^{s}(x) \quad \Leftrightarrow \quad \lim _{k \rightarrow \infty} v_{-}^{-k} \operatorname{dist}_{M}\left(f^{k}(y), f^{k}(x)\right)=0, \\
& y \in W^{u}(x) \Leftrightarrow \lim _{k \rightarrow \infty} v_{+}^{-k} \operatorname{dist}_{M}\left(f^{-k}(y), f^{-k}(x)\right)=0 .
\end{aligned}
$$

(b) If, in addition, $E^{0} \oplus E^{u}$ is integrable to a $C^{1}$-foliation and

- either $\mu_{-}>1$

- or $\mu_{-} \leq 1$ and (3.3) holds with $\delta=0$,

then $\nu_{-}$can be taken equal to $\min \left\{\mu_{-}, 1\right\}$. Similarly for $\nu_{+}$.

Proof. For $K=1,2, \ldots, \infty$, (a) follows from either [P] or [HPS, Theorem 5.5].

For $K=\omega$, one can use the approach in [HPS]. Endow $M$ with an analytic metric. Consider the complex analytic extension $\tilde{M}$ of $M$, endowed with a smooth extension of the Riemannian metric of $M$. Let $F: \tilde{M}_{0} \rightarrow \tilde{M}$ be the complex analytic extension of $f$, where $M \subset \tilde{M}_{0} \subset \tilde{M}, \tilde{M}_{0}$ open in $\tilde{M}$. The exponential map of $M$ also admits an analytic extension, hence we can lift $F$ to a complex analytic map $\hat{F}_{0}:\left(T_{\mathbb{C}} \tilde{M}_{0}\right)_{2 r} \rightarrow T_{\mathbb{C}} \tilde{M}$ for some small $r>0$ (by $E_{r}$ we denote the sub-bundle of balls of radius $r$ of the bundle $E$; $T_{\mathbb{C}} \tilde{M}$ denotes the tangent bundle of $\tilde{M}$ endowed with the natural complex structure, $J$ ). Viewing $E:=\left.T_{\mathbb{C}} \tilde{M}\right|_{M}$ as a real bundle only, extend $\left.\hat{F}_{0}\right|_{E_{2 r}}$ to a $C^{\infty}$ map $\hat{F}: E \rightarrow E$ which coincides with $\hat{F}_{0}$ on $E_{r}$ and is close to $\left.D F\right|_{E}$. Introduce $E_{\mathbb{C}}^{u}:=E^{u} \oplus J E^{u}$ and $E_{\mathbb{C}}^{c s}:=E^{c s} \oplus J E^{c s}$, where $E^{c s}:=E^{0} \oplus E^{s}$. Then $\left.D F\right|_{E}$ preserves the splitting $E=E_{\mathbb{C}}^{u} \oplus E_{\mathbb{C}}^{c s}$, and has similar exponential estimates as those of $D f$ for the splitting $T M=E^{u} \oplus E^{c s}$. Viewing $E$ as a real bundle, the conclusion of [HPS, $\left.\S 5\right]$ holds: see $E$ as a bundle over $E_{\mathbb{C}}^{u}$ via the projection along $E_{\mathbb{C}}^{c s}$, and consider in the space of sections the set

$$
\Gamma_{0}(1):=\left\{\sigma \in \Gamma\left(E_{\mathbb{C}}^{u}, E\right): \sigma\left(0_{p}\right)=0,\left\|\left.\sigma\right|_{E_{\mathbb{C}, p}^{u}}\right\|_{\text {Lip }} \leq 1 \text { for each } p \in M\right\}
$$

endowed with the metric $\left\|\sigma-\sigma^{\prime}\right\|_{*}:=\sup _{p \in M} \sup _{0 \neq x \in E_{\mathbb{C}, p}^{u}}\left\|\sigma(x)-\sigma^{\prime}(x)\right\| /\|x\|$. Then the graph transform $\hat{F}_{\#}$ is a contraction on $\Gamma_{0}(1)$ with respect to the metric $\|\cdot\|_{*}$, and the graph of the restriction of the invariant section $\sigma_{\hat{F}}$ to $E^{u}$ gives the exponential map via the unstable manifolds of $f$.

Let $\varepsilon>0$ be such that $\left(E_{\mathbb{C}}^{u}\right)_{\varepsilon} \oplus\left(E_{\mathbb{C}}^{c s}\right)_{\varepsilon} \subset\left(\left.T_{\mathbb{C}} \tilde{M}\right|_{M}\right)_{r}$. Consider the subset of $\Gamma_{0}(1)$ given by

$$
\begin{aligned}
\Gamma^{\omega}:=\left\{\sigma \in \Gamma_{0}(1): \sigma_{p, \varepsilon}\right. & \text { is complex analytic } \\
& \text { and depends continuously in } \left.C^{\omega} \text { on } p \in M\right\},
\end{aligned}
$$

where $\sigma_{p, \varepsilon}:=\left.\sigma\right|_{\left(E_{\mathbb{C}, p}^{u}\right)_{\varepsilon}}:\left(E_{\mathbb{C}, p}^{u}\right)_{\varepsilon} \rightarrow E_{\mathbb{C}, p}^{c s}$. Notice that $\Gamma^{\omega}$ is closed under $\|\cdot\|_{*}$, and it is easy to check that if $\hat{F}$ is close enough to $\left.D F\right|_{M}$ then $\Gamma^{\omega}$ is invariant under $\hat{F}_{\# \text {. }}$ Therefore $\sigma_{\hat{F}} \in \Gamma^{\omega}$, which yields the desired conclusion.

Case (b) is proven in [HPS, Corollary 5.6]. 
Remark. If the distribution $E^{s} \oplus E^{0}$ is integrable, the corresponding foliation is called 'center-stable' (similarly for 'center-unstable'). We will use these foliations only for $K=1,2, \ldots, \infty$ and either a flow or cases constructed as in the next theorem from an element of an $\mathcal{A}$-action, when their existence and smoothness are immediate.

The following theorem shows how cocycles are related to this situation. It is a straightforward extension of [BP, Theorem 2.2] (note that the hypothesis in the original version is equivalent to (3.5) by the spectral radius theorem).

THEOREM 3.2. Assume that $M$ is a compact manifold and $T \in \operatorname{Diff}^{K}(M)$ is a partially hyperbolic diffeomorphism, with Mather spectrum contained in the rings $\{z \in \mathbb{C}:|z| \geq$ $\left.\lambda_{+}\right\},\left\{z \in \mathbb{C}: \mu_{-} \leq|z| \leq \mu_{+}\right\}$and $\left\{z \in \mathbb{C}:|z| \leq \lambda_{-}\right\}$, where $0<\lambda_{-}<\mu_{-} \leq \mu_{+}<\lambda_{+}$, $\lambda_{-}<1<\lambda_{+}$. Let $F$ be a compact Riemannian manifold and $\beta: \mathbb{Z} \times M \rightarrow \operatorname{Diff}^{K}(F) a$ $C^{K}$ cocycle. Denote by $\tilde{T}$ the lifted action on $M \times F$, i.e. $\tilde{T}(x, y)=(T x, \beta(1, x)(y))$. If

$$
\begin{aligned}
& v_{+}:=\lim _{k \rightarrow \infty} \sup _{x \in M}\left\|D_{F} \beta(k, x)\right\|^{1 / k}<\lambda_{+}, \\
& v_{-}:=\lim _{k \rightarrow \infty} \sup _{x \in M}\left\|D_{F} \beta(k, x)^{-1}\right\|^{-1 / k}>\lambda_{-},
\end{aligned}
$$

then $\tilde{T}$ is a partially hyperbolic diffeomorphism.

Its spectrum is contained in the rings $\left\{z \in \mathbb{C}:|z| \geq \lambda_{+}\right\},\left\{z \in \mathbb{C}: \tilde{\mu}_{-} \leq|z| \leq \tilde{\mu}_{+}\right\}$ and $\left\{z \in \mathbb{C}:|z| \leq \lambda_{-}\right\}$, where $\tilde{\mu}_{+}=\max \left\{\mu_{+}, \nu_{+}\right\}, \tilde{\mu}_{-}=\min \left\{\mu_{-}, v_{-}\right\}$. The stable and unstable distributions of $\tilde{T}$ project through $D \pi$ onto those of $T$, hence the stable and unstable foliations project onto the stable, respectively unstable, foliations of $T$. The center distribution of $\tilde{T}$ is given by the preimage under $D \pi$ of the center distribution of $T$. If the center distribution of $T$ is integrable than so is that of $\tilde{T}$, and the center foliation of $\tilde{T}$ is given by the preimage under $\pi$ of the center foliation of $T$. In this case the center-stable and center-unstable foliations of $\tilde{T}$ are the preimages through $\pi$ of those of $T$.

\section{Limitations on regularity}

We are going to discuss an example due to de la Llave and a variant of it which shows that Theorems 2.2 and 2.4 are sharp as far as the Hölder assumption on the transfer map is concerned.

de la Llave used this counterexample in connection with the conjugacy of Anosov maps. Katok noticed that it can be used for the problem we are interested in. We will consider it from the viewpoint of partially hyperbolic maps, a class which includes cocycles close to the identity over Anosov actions (by Theorem 3.2). We will first describe the example, relate it to partially hyperbolic maps and make some remarks. We will then construct a similar example for the case of Lie-group valued cocycles (although the original example can be seen as taking values in the group of affine transformations, it does not give the bound we need).

Consider a linear hyperbolic $\mathbb{Z}$-action on $\mathbb{T}^{2}$ given by $A \in \mathrm{SL}(2, \mathbb{Z})$ and a constant cocycle $\beta(1, \cdot) \equiv B \in \operatorname{Diff}\left(\mathbb{T}^{d}\right)$, where $B \in \operatorname{SL}(d, \mathbb{Z}), d \geq 2$. 
THEOREM 4.1. (de la Llave [Lla2, Theorem 6.3]) For a dense set of values $r \in(0, \infty)$ there is a choice of $A$ and $B$ for which there are arbitrarily $C^{\infty}$-small smooth perturbations $\widetilde{\beta}$ of $\beta$ with the property that for any $\varepsilon>0$ the cocycles $\beta$ and $\widetilde{\beta}$ are cohomologous by a $C^{r-\varepsilon}$ transfer map, but not by a $C^{r+\varepsilon}$ transfer map.

Assume that $A \in \mathrm{SL}(2, \mathbb{Z})$ and $B \in \mathrm{SL}(d, \mathbb{Z})$ are hyperbolic matrices such that $B$ has a real eigenvalue in the interval $(1, \infty)$. Let $0<\lambda<1$ be one of the eigenvalues of $A$ (hence the other one is $\lambda^{-1}$ ), and let $\mu>1$ be a real eigenvalue of $B$. We denote the corresponding normalized eigenvectors as

$$
\begin{aligned}
A \mathbf{v}_{-} & =\lambda \mathbf{v}_{-}, \\
A \mathbf{v}_{+} & =\lambda^{-1} \mathbf{v}_{+}, \\
B \mathbf{e}_{\mu} & =\mu \mathbf{e}_{\mu} .
\end{aligned}
$$

Consider a function $\varphi: \mathbb{T}^{2} \rightarrow \mathbb{R}$ and the following actions on $\mathbb{T}^{2} \times \mathbb{T}^{d}$ :

$$
\begin{gathered}
f(x, y)=(A x, B y), \\
\tilde{f}(x, y)=\left(A x, B y+\varphi(x) \mathbf{e}_{\mu}\right) .
\end{gathered}
$$

(We see the quantity $\varphi(x) \mathbf{e}_{\mu}$ as its image in $\mathbb{T}^{d}=\mathbb{R}^{d} / \mathbb{Z}^{d}$, which is an abelian group.)

Note that $f$ is hyperbolic, hence, for $C^{1}$-small $\varphi, \widetilde{f}$ is also hyperbolic and there is a homeomorphism $h \in \operatorname{Homeo}\left(\mathbb{T}^{2} \times \mathbb{T}^{d}\right)$ close to identity such that

$$
h \widetilde{f}=f h .
$$

Moreover, this homeomorphism is unique among the homeomorphisms which are homotopic to identity. The unique solution to (4.1) homotopic to identity is given by

$$
h(x, y)=\left(x, y+\psi(x) \mathbf{e}_{\mu}\right),
$$

provided that $\psi: \mathbb{T}^{2} \rightarrow \mathbb{R}$ satisfies the equation

$$
\mu \psi(x)-\psi(A x)=\varphi(x) .
$$

Equation (4.2) admits a unique bounded solution, namely

$$
\psi(x)=\mu^{-1} \sum_{k=0}^{\infty} \mu^{-k} \varphi\left(A^{k} x\right) .
$$

It remains to find out how smooth $\psi$ is, assuming that $\varphi$ is $C^{\infty}$.

Let

$$
\alpha_{C}:=\frac{\ln \mu}{\ln \lambda^{-1}} .
$$

It can be proven by a straightforward computation that $\psi$ is of class $C^{\alpha}$ for any $\alpha<\alpha_{C}$.

By choosing $\varphi$ to be a trigonometric polynomial and using Fourier series, de la Llave shows that

$$
\psi \notin C^{r}, \quad \text { for any } r>\alpha_{C} .
$$

Since multiplying $\varphi$ by a constant changes $\psi$ by the same factor, we see that the perturbation can be made as small as desired, while preserving the loss of regularity. 
Note also that $\alpha_{C}$ can take a dense set of values in $(0, \infty)$ if we replace $A$ and $B$ by their powers.

We can see $f$ and $\tilde{f}$ as $\operatorname{Diff}\left(\mathbb{T}^{d}\right)$-valued cocycles over the hyperbolic action $x \in$ $\mathbb{T}^{2} \mapsto A x \in \mathbb{T}^{2} . f$ corresponds to $\beta(1, x)(y)=B y$ and $\widetilde{f}$ to

$$
\widetilde{\beta}(1, x)(y)=B y+\varphi(x) \mathbf{e}_{\mu} .
$$

Since $h^{-1}$ is a bundle map, it corresponds to a transfer map between $\beta$ and $\widetilde{\beta}$ given by

$$
x \in \mathbb{T}^{2} \mapsto P_{x}(\cdot)=\cdot-\psi(x) \mathbf{e}_{\mu} \in \operatorname{Diff}^{\infty}\left(\mathbb{T}^{d}\right),
$$

i.e.

$$
\tilde{\beta}(g, x)=P_{g x} \circ B \circ P_{x}^{-1} .
$$

This clarifies Theorem 4.1.

Let us now assume that $\left\|B^{ \pm 1}\right\|<\lambda^{-1}$. Then $f$ and $\tilde{f}$ become partially hyperbolic diffeomorphisms (by Theorem 3.2).

Notice that in this case the critical value $\alpha_{C}<1$. Hence $P$ fails to be $C^{1}$.

Remark. In the example considered above, if the spectrum of $B$ is contained in the ring $\left\{z \in \mathbb{C}: \mu^{-1} \leq|z| \leq \mu\right\}$, then the critical order $\alpha_{C}$ where $P$ fails to be Hölder is exactly the value $\alpha_{0}$ of Theorem 2.2.

Given a partially hyperbolic diffeomorphism of a compact manifold, by a horizontal foliation we mean a $C^{1}$-foliation which is transverse and complementary to the neutral distribution. Note that any $C^{1}$ invariant horizontal foliation has to contain (and hence, is spanned by) the stable and unstable foliations (because the only invariant distribution which is not tangent to the center one has to be contained in $E^{s} \oplus E^{u}$ ).

But any (invariant) $C^{0}$ foliation spanned by the stable and unstable foliations of a $C^{r}$ partially hyperbolic diffeomorphism is actually $C^{r-\varepsilon}$, because Journé's Theorem 5.1 implies that if two uniformly $C^{r}$ foliations $\mathcal{F}_{1}$ and $\mathcal{F}_{2}$ span a $C^{0}$-foliation $\mathcal{F}$, then the latter is $C^{r-\varepsilon}$. Indeed, consider a leaf $\mathcal{L}$ of $\mathcal{F}$; choose smooth local coordinates in such a way that a small open set of $\mathcal{L}$ can be seen as the graph of a function $\phi: U \rightarrow V$ defined on a small domain $U$ whose tangent space is close to the span of the tangent spaces of $\mathcal{F}_{1}$ and $\mathcal{F}_{2}$. Since $\phi$ is uniformly $C^{r}$ along each of the two transversal foliations obtained by projecting $\mathcal{F}_{1} \cap \mathcal{L}$ and $\mathcal{F}_{2} \cap \mathcal{L}$ along $V$ to $U$, we conclude that $\phi$ is $C^{r-\varepsilon}$.

Since $\beta$ and $\tilde{\beta}$ are cohomologous, one obtains an invariant $C^{0}$-foliation for $\tilde{\beta}$ (more precisely, for $\tilde{f}$ ) by taking the image under $\mathbf{P}$ of the horizontal invariant foliation of $f$. The horizontal invariant foliation of $f$ has leaves $\mathcal{L}(x, y)=\mathbb{T}^{2} \times\{y\},(x, y) \in \mathbb{T}^{2} \times \mathbb{T}^{d}$. Its image under $\mathbf{P}$ has leaves $\tilde{\mathcal{L}}(x, y)=\left\{\left(z, P_{z} \circ P_{x}^{-1}(y)\right): z \in \mathbb{T}^{2}\right\}$.

Since $P$ is not $C^{1}, \tilde{\mathcal{L}}(x, y)$ is not $C^{1}$ either, hence it cannot contain the leaves of $\tilde{f}$. Indeed, one computes that the $D \tilde{f}$ invariant splitting $E_{\tilde{f}}^{s} \oplus E^{0} \oplus E_{\tilde{f}}^{u}$ of $T\left(\mathbb{T}^{2} \times \mathbb{T}^{d}\right) \cong$ $T \mathbb{T}^{2} \times T \mathbb{T}^{d}$ is given by

$$
\begin{aligned}
E_{\tilde{f}}^{s}(x, y) & =\mathbb{R}\left(\mathbf{v}_{-}, \rho^{s}(x) \mathbf{e}_{\mu}\right), \quad E_{\tilde{f}}^{u}(x, y)=\mathbb{R}\left(\mathbf{v}_{+}, \rho^{u}(x) \mathbf{e}_{\mu}\right), \\
\rho^{s}(x): & =-\mu^{-1}\left[\sum_{k=0}^{\infty}\left(\frac{\lambda}{\mu}\right)^{k} D \varphi_{A^{k} x}\left(\mathbf{v}_{-}\right)\right]
\end{aligned}
$$




$$
\rho^{u}(x):=\lambda\left[\sum_{k=0}^{\infty}(\lambda \mu)^{k} D \varphi_{A^{-k-1} x}\left(\mathbf{v}_{+}\right)\right] .
$$

Note that the distribution $E_{\tilde{f}}^{s}$ is transversally $C^{1}$. The stable leaves are

$$
\begin{gathered}
W_{\tilde{f}}^{s}(x, y)=\left\{\left(x+t \mathbf{v}_{-}, y+\omega_{x, y}^{s}(t) \mathbf{e}_{\mu}\right): t \in \mathbb{R}\right\}, \\
\omega_{x, y}^{s}(t):=-\mu^{-1} \sum_{k=0}^{\infty} \mu^{-k}\left[\varphi\left(A^{k}\left(x+t \mathbf{v}_{-}\right)\right)-\varphi\left(A^{k}(x)\right)\right],
\end{gathered}
$$

and the unstable leaves are

$$
\begin{gathered}
W_{\tilde{f}}^{u}(x, y)=\left\{\left(x+t \mathbf{v}_{+}, y+\omega_{x, y}^{u}(t) \mathbf{e}_{\mu}\right): t \in \mathbb{R}\right\}, \\
\omega_{x, y}^{u}(t):=\sum_{k=0}^{\infty} \mu^{k}\left[\varphi\left(A^{-k-1}\left(x+t \mathbf{v}_{+}\right)\right)-\varphi\left(A^{-k-1}(x)\right)\right] .
\end{gathered}
$$

Hence $\mathbf{P}\left(W_{f}^{u}(x, y)\right) \nsubseteq W_{\tilde{f}}^{u}(\mathbf{P}(x, y))$, but $\mathbf{P}\left(W_{f}^{s}(x, y)\right) \subset W_{\tilde{f}}^{s}(\mathbf{P}(x, y))$ ! Note that $\left.P\right|_{W_{A}^{s}}$ is as smooth as $\varphi$, and the breakdown in the smoothness of $P$ occurs along $W_{A}^{u}$. These facts are in agreement with the results to be discussed next.

We turn now to the case of Lie-group valued cocycles. We describe only the simplest situation, taking values in $\operatorname{GL}(2, \mathbb{R})$.

THEOREM 4.2. Let $A \in \mathrm{SL}(2, \mathbb{Z})$ be a hyperbolic matrix with eigenvalues $\lambda^{ \pm 1}, 0<\lambda<1$, which acts on $\mathbb{T}^{2}$ and $\beta$ the constant cocycle given by

$$
\beta(1, \cdot) \equiv B:=\left(\begin{array}{cc}
\mu & 0 \\
0 & \nu
\end{array}\right)
$$

where $0<v<\mu$. Let $r:=\ln \left(\mu v^{-1}\right) / \ln \lambda^{-1}$.

There are arbitrarily $C^{\infty}$-small smooth perturbations $\widetilde{\beta}(1, \cdot): \mathbb{T}^{2} \rightarrow \mathrm{GL}(2, \mathbb{R})$ of $\beta$ with the property that for any $\varepsilon>0$ the cocycles $\beta$ and $\widetilde{\beta}$ are cohomologous by a $C^{r-\varepsilon}$ transfer map, but not by a $C^{r+\varepsilon}$ transfer map.

Proof. Consider $\widetilde{\beta}$ and $P$ given by

$$
\widetilde{\beta}(1, x):=\left(\begin{array}{cc}
\mu & \varphi(x) \\
0 & v
\end{array}\right), \quad P(x):=\left(\begin{array}{cc}
1 & \psi(x) \\
0 & 1
\end{array}\right) .
$$

The cohomology equation (2.1) is equivalent to

$$
\left(\mu v^{-1}\right) \psi(x)-\psi(A x)=v^{-1} \varphi(x),
$$

and by the same arguments as before it admits a solution $\psi: \mathbb{T}^{2} \rightarrow \mathbb{R}$ of class $C^{r-\varepsilon}$, provided $\varphi$ is smooth enough. Moreover, by the argument of de la Llave, we can choose a smooth function $\varphi$ such that (4.3) does not admit solutions $\psi$ of class $C^{r+\varepsilon}$ for any $\varepsilon>0$.

It remains to show that no other transfer map between $\beta$ and $\widetilde{\beta}$ can have a higher regularity. This follows from the fact that the transfer map is essentially unique. 
Assume that $Q: \mathbb{T}^{2} \rightarrow \mathrm{GL}(2, \mathbb{R})$ is continuous and $\beta(n, x)=Q\left(A^{n} x\right) \tilde{\beta}(n, x) Q(x)^{-1}$. Let $R(x):=P(x) Q(x)^{-1}$, where $P$ is the transfer map found above. Then $\beta(n, x)=$ $R\left(A^{n} x\right) \beta(n, x) R(x)^{-1}$, i.e.

$$
R(x)=B^{-n} R\left(A^{n} x\right) B^{n} .
$$

But for $R$ continuous this can happen only if $R$ is a constant diagonal matrix. Indeed, if we write

$$
R(x)=\left(\begin{array}{ll}
s(x) & t(x) \\
u(x) & v(x)
\end{array}\right)
$$

then (4.4) implies

$$
\begin{gathered}
s(x)=s\left(A^{n} x\right), \quad t(x)=\left(\mu^{-1} v\right)^{n} t\left(A^{n} x\right), \\
u(x)=\left(\mu^{-1} v\right)^{n} u\left(A^{-n} x\right), \quad v(x)=v\left(A^{n} x\right),
\end{gathered}
$$

which shows that $t \equiv 0, u \equiv 0$ and $s, v$ are constant (because $A$ is transitive).

Thus we see that in Theorem 2.4 one indeed needs a more restrictive assumption on the transfer map than in Theorem2.2.

5. Proof of Theorem 2.2 for $1 \leq K \leq \infty$

For the proof we need the following.

THEOREM 5.1. (Journé $[\mathbf{J}]$ ) Assume on a manifold that we are given two continuous transverse foliations, $F_{s}$ and $F_{u}$, with uniformly smooth leaves. If the function $f$ is uniformly $C^{K+\alpha}$-smooth along the leaves of $F_{s}$ and $F_{u}$, then $f$ is $C^{K+\alpha}$-smooth $(1 \leq K \leq \infty, \alpha \in(0,1))$.

The idea of the proof of Theorem 2.2 is the following. The condition $\lambda_{-}<\mu_{-} \leq$ $\mu_{+}<\lambda_{+}$implies that the lifts $f, \tilde{f} \in \operatorname{Diff}^{K}(M \times N)$ of $T^{1}$ corresponding to $\beta(1, \cdot)$ and $\tilde{\beta}(1, \cdot)$ are partially hyperbolic diffeomorphisms. The Hölder assumption on $P$ implies that the conjugacy $(x, y) \in M \times N \stackrel{P}{\mapsto}\left(x, P_{x}(y)\right)$ maps the stable and unstable foliations of $\tilde{f}$ into those of $f$. Due to the special form of $f$ and $\tilde{f}$, this implies that $\mathbf{P}$ is $C^{K}$ along the foliations of $\tilde{f}$. The smoothness along the center foliation follows easily from the hypotheses-invoking Journé's theorem for the case of a flow-and repeated use of this theorem gives the desired conclusion.

Note that one can apply this approach to a conjugacy between two partially hyperbolic diffeomorphisms having integrable center distributions (say, with compact leaves). The following conditions are required: that the neutral foliations be transversally smooth, that they be mapped one onto the other by the conjugacy, and that the two Anosov maps induced on the quotient spaces be smoothly conjugated. However, one can obtain a better result for actions of $\mathbb{Z}^{k}, k \geq 2$, if the stable foliations produce a 'trellis' of one-dimensional leaves which is complementary to the neutral foliation (see $[\mathbf{H}]$ or $[\mathbf{K L}]$ for the definition of a trellis). Such an argument is used in [NT1, §7].

Remark. The difference between this approach and the previous results [LMM, Lla1, NT2] is the following. In the cited papers one proved regularity results for cohomologically trivial cocycles by exhibiting the derivative of the transfer map ( $P$ in 
our notation) along the horizontal lifts of the stable and unstable foliations of the action on the base ( $T^{t}$ in our case). The same computation in the case considered here does not seem to lead to a convergent expression, even with the Hölder assumption. As one can see, the directions along which the derivatives are natural are the stable and unstable leaves of the lifted action (which are indeed horizontal for the trivial cocycle). The computational part is taken care of by Theorem 3.1(a), respectively Theorem A.1.

Let us now give the details of the proof.

Denote the $\mathcal{A}$-invariant splitting of $T M$ by $E^{s} \oplus E^{0} \oplus E^{u}$, and the corresponding foliations of $T^{1}$ by $W_{0}^{s}, W_{0}^{c}$ and $W_{0}^{u}$, respectively.

Consider $f, \tilde{f} \in \operatorname{Diff}^{K}(M \times N)$ given by $f(x, y):=\left(T^{1} x, \beta(1, x)(y)\right)$, respectively $\tilde{f}(x, y):=\left(T^{1} x, \tilde{\beta}(1, x)(y)\right)$. By Theorem 3.2, $f$ and $\tilde{f}$ are partially hyperbolic. Denote the $D f$-invariant splitting of $T(M \times N) \cong T M \times T N$ by $F^{s} \oplus F^{0} \oplus F^{u}$, and the stable and unstable foliations of $f$ by $W^{s}$, respectively $W^{u}$. The corresponding notations for $\tilde{f}$ are $\tilde{F}^{s} \oplus F^{0} \oplus \tilde{F}^{u}, \tilde{W}^{s}$, respectively $\tilde{W}^{u}$.

Note that $F^{0}=\{0\} \times T N \oplus E^{0} \times\{0\}$. Hence there is a constant $C$ such that for each $k$

$$
\left\|\left.D f^{k}\right|_{F^{0}}\right\| \leq C\left(\sup _{x \in M}\left\|D_{N} \beta(k, x)\right\|+\sup _{x \in M}\left\|\left.D T^{k}\right|_{E_{0}}\right\|\right),
$$

and similarly for $\tilde{f}$. The second term in the above sum is bounded uniformly with respect to $k$ because $D T$ preserves the vector field which generates the flow.

Consider $\mathbf{P} \in \operatorname{Homeo}(M \times N)$ given by $\mathbf{P}(x, y):=\left(x, P_{x}(y)\right)$, where $P_{x}=P(x)$. Then $f \mathbf{P}=\mathbf{P} \tilde{f}$, because $P$ is a transfer map between $\beta$ and $\tilde{\beta}$.

It is immediate that if $P: M \times N \rightarrow N$ is $\alpha$-Hölder then the same holds for $\mathbf{P}$. We claim that the fact that $P: M \times N \rightarrow N$ is $\alpha$-Hölder implies that $P^{-1}: M \times N \rightarrow N$, and therefore $\mathbf{P}^{-1}$, are also $\alpha$-Hölder. Indeed, notice that in the $N$-variable $P: M \times N \rightarrow N$ is $C^{1}$, hence Lipschitz. This property remains true for $P^{-1}: M \times N \rightarrow N$. We only have to check the Hölder property in the $M$-variable. For $x^{\prime}, x^{\prime \prime} \in M$ and $y \in N$ one has

$$
\begin{aligned}
\operatorname{dist}_{N}\left(P_{x^{\prime}}^{-1}(y), P_{x^{\prime \prime}}^{-1}(y)\right) & =\operatorname{dist}_{N}\left(P_{x^{\prime}}^{-1}\left(P_{x^{\prime \prime}}\left(P_{x^{\prime \prime}}^{-1}(y)\right)\right), P_{x^{\prime}}^{-1}\left(P_{x^{\prime}}\left(P_{x^{\prime \prime}}^{-1}(y)\right)\right)\right) \\
& \leq \operatorname{Lip}\left(P_{x^{\prime}}^{-1}\right) \operatorname{dist}_{N}\left(P_{x^{\prime \prime}}\left(P_{x^{\prime \prime}}^{-1}(y)\right), P_{x^{\prime}}\left(P_{x^{\prime \prime}}^{-1}(y)\right)\right) \\
& \leq \operatorname{Lip}\left(P_{x^{\prime}}^{-1}\right) \cdot C \cdot\left[\operatorname{dist}_{M}\left(x^{\prime}, x^{\prime \prime}\right)\right]^{\alpha} .
\end{aligned}
$$

By Theorem 3.2, the Mather spectra of $f$ and $\tilde{f}$ are contained in the rings bounded by the circles of radii 0 and $\lambda_{-}, \mu_{-}$and $\mu_{+}$, respectively $\lambda_{+}$and $\infty$. Their stable foliations are characterized by Theorem 3.1. Let $z \in \tilde{W}^{s}(u), u, z \in M \times N$. For $\tilde{v}_{-}>\lambda_{-}$one has

$$
\lim _{k \rightarrow \infty} \tilde{v}_{-}^{-k} \operatorname{dist}_{M \times N}\left(\tilde{f}^{k}(z), \tilde{f}^{k}(u)\right)=0 .
$$

Assume that $P$ is $\alpha$-Hölder for some $\alpha>0$. Since

$$
\begin{aligned}
\operatorname{dist}_{M \times N}\left(f^{k}(\mathbf{P}(z)), f^{k}(\mathbf{P}(u))\right) & =\operatorname{dist}_{M \times N}\left(\mathbf{P}\left(\tilde{f}^{k}(z)\right), \mathbf{P}\left(\tilde{f}^{k}(u)\right)\right) \\
& \leq C^{\prime}\left[\operatorname{dist}_{M \times N}\left(\tilde{f}^{k}(z), \tilde{f}^{k}(u)\right)\right]^{\alpha},
\end{aligned}
$$

it follows that

$$
\lim _{k \rightarrow \infty} \tilde{v}_{-}^{-\alpha k} \operatorname{dist}_{M \times N}\left(f^{k}(\mathbf{P}(z)), f^{k}(\mathbf{P}(u))\right)=0 .
$$


By Theorem 3.1(a), this implies that $\mathbf{P}(z) \in W^{s}(\mathbf{P}(u))$, provided that $\tilde{v}_{-}^{\alpha}<\mu_{-}$. If $\alpha>\ln \mu_{-} / \ln \lambda_{-}$one can find a $\tilde{v}_{-}>\lambda_{-}$which satisfies this condition, thus proving that $\mathbf{P}\left(\tilde{W}^{s}(u)\right) \subset W^{s}(\mathbf{P}(u))$.

Assume now that $\alpha_{0}=0$ and $P: M \times N \rightarrow N$ is only continuous (without any Hölder assumption). Then instead of (5.1) we obtain only that

$$
\lim _{k \rightarrow \infty} \operatorname{dist}_{M \times N}\left(f^{k}(\mathbf{P}(z)), f^{k}(\mathbf{P}(u))\right)=0 .
$$

However, by our additional hypothesis,

$$
\lim _{k \rightarrow \infty}\left\|\left(\left.D f^{-k}\right|_{F^{0}}\right)\right\|<\infty,
$$

hence Theorem 3.1(b) implies that $\mathbf{P}(z) \in W^{s}(\mathbf{P}(u))$, as desired.

In conclusion, we have proved that under the hypotheses of the theorem $\mathbf{P}\left(\tilde{W}^{s}(u)\right) \subset$ $W^{s}(\mathbf{P}(u))$.

However, both $\tilde{W}^{s}(u)$ and $W^{s}(\mathbf{P}(u))$ are graphs of some functions $\tilde{\gamma}_{(x, y)}, \gamma_{(x, P(x, y))}$ : $W_{0}^{s}(x) \rightarrow N$, where $u=(x, y) \in M \times N$ and $\tilde{\gamma}_{(x, y)}(x)=y, \gamma_{(x, P(x, y))}(x)=$ $P(x, y)$. Since $\mathbf{P}$ preserves each vertical fiber, the inclusion has to be equality, and $P\left(t, \tilde{\gamma}_{u}(t)\right)=\gamma_{\mathbf{P}(u)}(t)$, for all $t \in W_{0}^{s}(x)$. Since the foliations $W^{s}$ and $\tilde{W}^{s}$ are $C^{K}$ (in the sense specified in $\S 3$ ), it follows that $\gamma_{(x, y)}, \tilde{\gamma}_{(x, P(x, y))}$ are $C^{K}$ functions, whose derivatives vary continuously with $(x, y) \in M \times N$ and $t \in W_{0}^{s}(x)$. From the inverse function theorem applied to $t \in W_{0}^{s}(x) \mapsto\left(t, \tilde{\gamma}_{u}(t)\right) \in \tilde{W}^{s}(u)$, one obtains that $\left.\mathbf{P}\right|_{\tilde{W}^{s}(u)}: \tilde{W}^{s}(u) \rightarrow W^{s}(\mathbf{P}(u)) \subset M \times N$ is $C^{K}$, with the same continuous dependence. Note that we have a similar statement for $\left.\mathbf{P}^{-1}\right|_{W^{s}(\mathbf{P}(u))}: W^{s}(\mathbf{P}(u)) \rightarrow \tilde{W}^{s}(u)$.

We proved that $\mathbf{P}$ is uniformly $C^{K}$ along $\tilde{W}^{s}$, and $\mathbf{P}^{-1}$ is uniformly $C^{K}$ along $W^{s}$. Repeating the proof for $f^{-1}$ and $\tilde{f}^{-1}$ we obtain that $\mathbf{P}$ is uniformly $C^{K}$ along $\tilde{W}^{u}$, and $\mathbf{P}^{-1}$ is uniformly $C^{K}$ along $W^{u}$.

The center foliation $W^{c}$ of both $f$ and $\tilde{f}$ is spanned by the transverse foliations $W_{v}^{c}$ and $W_{h}^{c}$ corresponding to the vertical fibers, respectively the center foliation of $T^{1}$ : the former is tangent to $\{0\} \times T N$, the latter to $E^{0} \times\{0\}$. $\mathbf{P}$ is uniformly $C^{K}$ along $W_{v}^{c}$ by the hypothesis. For the case of a flow, the cohomology equation (2.1) gives

$$
P\left(T^{t}(x), y\right)=\beta(t, x) \circ P_{x} \circ \tilde{\beta}(t, x)^{-1}(y)
$$

which shows that $\mathbf{P}$ is uniformly $C^{K}$ along $W_{h}^{c}$ as well. Since $W^{c}$ and $\tilde{W}^{s}$ can be integrated into the center-stable foliation and the latter together with $\tilde{W}^{u}$ span the whole manifold $M \times N$, applying Journé's Theorem 5.1 successively we obtain that $\mathbf{P}$ is $C^{K-\varepsilon}$ for any small $\varepsilon>0$. Note that if $K=1$ then there is no need for Theorem 5.1, hence no loss of regularity occurs.

\section{The analytic case}

The reason we have to consider this case separately is that Journé's Theorem 5.1 does not include the $C^{\omega}$ case. The alternative approach is to estimate the decay of Fourier coefficients. This was done in [Lla1]. The following is a paraphrase of [Lla1, Lemma 2.5]. Its proof is the same as for the original statement. 
THEOREM 6.1. (de la Llave [Lla1]) Assume that the real-analytic compact manifold $X$ is spanned by a family of $C^{\omega}$-foliations $\mathcal{F}_{1}, \mathcal{F}_{2}, \ldots, \mathcal{F}_{k}$ with the property that the Jacobian $J_{i}$ of $\mathcal{F}_{i}$ is in $C_{\mathcal{F}_{i}}^{\omega}(X, \mathbb{R}), i=1, \ldots, k$. Then

$$
\bigcap_{i=1}^{k} C_{\mathcal{F}_{i}}^{\omega}(X, \mathbb{R})=C^{\omega}(X, \mathbb{R}) .
$$

Note that this is a local result, and this is how we are going to use it.

The Jacobian of a foliation is defined in Theorem 6.4. By $C_{\mathcal{F}}^{\omega}(X, \mathbb{R})$ we mean functions which are analytic along the leaves of the foliation $\mathcal{F}$ and depend continuously in the $C^{\omega}$-topology on the point of the manifold through which the (local) leaf is considered. (See Definition 6.2 for the description of the $C^{\omega}$-topology.)

In order to use Theorem 6.1, we have to show that the Jacobians of the stable/unstable foliations of an analytic partially hyperbolic diffeomorphism are analytic along the leaves. This was done for a flow in [Lla1] using the set-up of Anosov [A] and some results of [LMM]. We are going to follow the paper of Pugh and Shub [PS], where an expression for the Jacobian is given. We obtain expressions for the Jacobian and its derivative along the leaf which are counterparts of the infinitesimal versions found in [LMM]. These are (6.3) and (6.5). Although here we consider the $C^{\omega}$ case, these formulae hold whenever the partially hyperbolic diffeomorphism is at least $C^{2}$. From (6.5) one can obtain the higher derivatives by a straightforward differentiation.

Let us first recall the precise definitions.

Definition 6.2. Let $U \subset \mathbb{R}^{n}$ be an open set. The (vector) space $C^{\omega}\left(U, \mathbb{R}^{\ell}\right)$ of analytic mappings from $U$ to $\mathbb{R}^{\ell}$ is topologized by the following system of neighborhoods of the origin: $f$ is in $\mathcal{V}_{r, L}$ if it admits a complex-analytic extension $\tilde{f}: U_{r} \rightarrow \mathbb{C}^{\ell}$ such that $\sup _{\mathbf{z} \in U_{r}}\|f(\mathbf{z})\|<L$, where $U_{r}:=\left\{\mathbf{z}=\left(z_{1}, z_{2}, \ldots, z_{n}\right) \in \mathbb{C}^{n}:\left|\operatorname{Im} z_{i}\right| \leq r\right.$, $\left.\left(\operatorname{Re} z_{1}, \operatorname{Re} z_{2}, \ldots, \operatorname{Re} z_{n}\right) \in U\right\}$.

Assume that $X$ is a compact analytic manifold, with a $C^{\omega}$ Riemannian metric. Consider a partially hyperbolic diffeomorphism $f \in \operatorname{Diff}^{\omega}(X)$. Denote the splitting of $T X$ by $T X=E^{u} \oplus E^{c s}$ where $E^{c s}=E^{0} \oplus E^{s}$, and the unstable foliation by $W^{u}$. By Theorem 3.1(a), $W^{u}$ is a $C^{\omega}$-foliation.

Definition 6.3 Given a $C^{K}$-foliation $(K=1,2, \ldots, \omega)$, by a $C^{K}$ foliated chart we mean a map $\chi: U \times V \rightarrow X$ which is a homeomorphism onto an open subset of $X$ and such that, for each $v \in V, \chi_{v}:=\chi(\cdot, v): U \rightarrow X$ describes locally a leaf of the foliation, $\chi_{v}$ is $C^{K}$, and depends continuously on $v \in V$ in the $C^{K}$-topology.

Let $\mu$ be the measure induced by the Riemannian metric on $X$.

THEOREM 6.4. Given any point $x_{0} \in X$, there is a $C^{\omega}$ foliated chart $\chi: U \times V \rightarrow X$ of $W^{u}$ around $x_{0}$ such that $\chi^{*} \mu=\rho(t, v) d t d v$, with the Jacobian $\rho$ continuous on $U \times V$, analytic in the $U$-variable, and such that $v \in V \mapsto \rho(\cdot, v) \in C^{\omega}(U)$ is continuous.

Proof. Let $\Omega^{\prime}$ and $\Omega^{\prime \prime}$ be two smooth transversals to $W^{u}$, and denote by $h: \Omega^{\prime} \rightarrow \Omega^{\prime \prime}$ the holonomy along $W^{u}$. Pugh and Shub [PS, Theorem 2.1] showed that $h$ is absolutely 
continuous with respect to the measure induced on the transversals by the Riemannian metric. The Radon-Nikodym derivative is given by

$$
J_{\Omega^{\prime \prime}}^{\Omega^{\prime}}(y)=\lim _{n \rightarrow \infty} \frac{\operatorname{det}\left(f^{-n} \mid T_{y} \Omega^{\prime}\right)}{\operatorname{det}\left(f^{-n} \mid T_{h(y)} \Omega^{\prime \prime}\right)}, \quad y \in \Omega^{\prime},
$$

the limit being uniform.

Here by $\operatorname{det}(f \mid V)$, where $V \subset T_{x} X$ is a vector space, we mean the absolute value of the determinant of $\left.D f_{x}\right|_{V}$ with respect to a pair of orthonormal bases of $V \subset T_{x} X$, respectively $D_{x} f(V) \subset T_{f(x)} X$.

We want to show that this Jacobian is $C^{\omega}$ along the leaves of $W^{u}$.

First we have to write (6.1) in a different form. This expression of the Jacobian is valid whenever (6.1) holds.

Choose a continuous bundle $\tilde{F}$ which is complementary to $E^{u}$. Denote by $\tilde{\pi}: T X \rightarrow$ $\tilde{F}$ the projection along $E^{u}$. Denote by $\tilde{f}:=\left.\tilde{\pi} \circ D f\right|_{\tilde{F}}$ the compression of $D f$ to $\tilde{F}$. Since $\tilde{f}$ preserves $E^{u}$, the matrix of $D f$ with respect to the decomposition $T X=E^{u} \oplus \tilde{F}$ has the form

$$
D f=\left(\begin{array}{cc}
* & * \\
0 & \tilde{f}
\end{array}\right) .
$$

Note that $\tilde{f}$ is an invertible bundle map on $\tilde{F}$.

CLAIM 1.

$$
J_{\Omega^{\prime \prime}}^{\Omega^{\prime}}(y)=\lim _{n \rightarrow \infty} \frac{\operatorname{det}\left(\tilde{f}^{-n} \mid \tilde{F}_{y}\right)}{\operatorname{det}\left(\tilde{f}^{-n} \mid \tilde{F}_{h(y)}\right)} \cdot \frac{\operatorname{det}\left(\tilde{\pi} \mid T_{y} \Omega^{\prime}\right)}{\operatorname{det}\left(\tilde{\pi} \mid T_{h(y)} \Omega^{\prime \prime}\right)}, \quad y \in \Omega^{\prime},
$$

the limit being uniform.

Proof. Denote by $\pi: T X \rightarrow E^{c s}$ the projection along $E^{u}$. Then

$$
J_{\Omega^{\prime \prime}}^{\Omega^{\prime}}(y)=\lim _{n \rightarrow \infty} \frac{\operatorname{det}\left(f^{-n} \mid E_{y}^{c s}\right)}{\operatorname{det}\left(f^{-n} \mid E_{h(y)}^{c s}\right)} \cdot \frac{\operatorname{det}\left(\pi \mid T_{y} \Omega^{\prime}\right)}{\operatorname{det}\left(\pi \mid T_{h(y)} \Omega^{\prime \prime}\right)}, \quad y \in \Omega^{\prime},
$$

the limit being uniform. Note that this is equivalent to (6.1): $\pi$ commutes with $D f$, hence $\left.D f^{-n}\right|_{T_{y} \Omega^{\prime}}=\left.\left.\left(\left.\pi\right|_{T_{f-n(v)}} f^{-n}\left(\Omega^{\prime}\right)\right)^{-1} \circ D f^{-n}\right|_{E_{y}^{c s}} \circ \pi\right|_{T_{y} \Omega^{\prime}}$, which implies that $\operatorname{det}\left(f^{-n} \mid T_{y} \Omega^{\prime}\right)=\operatorname{det}\left(f^{-n} \mid E_{y}^{c s}\right) \operatorname{det}\left(\pi \mid T_{y} \Omega^{\prime}\right) / \operatorname{det}\left(\pi \mid T_{f^{-n}(y)} f^{-n}\left(\Omega^{\prime}\right)\right)$, and the denominator converges uniformly to 1 as $n \rightarrow \infty$. See [PS, formula $\left(\mathrm{b}^{\prime \prime}\right)$ ].

Since $\left.\tilde{f}^{k} \circ \tilde{\pi}\right|_{E^{c s}}=\left.\tilde{\pi} \circ D f^{k}\right|_{E^{c s}}$, one obtains that

$$
\operatorname{det}\left(f^{-n} \mid E_{z}^{c s}\right)=\operatorname{det}\left(\tilde{f}^{-n} \mid \tilde{F}_{z}\right) \operatorname{det}\left(\tilde{\pi}_{z} \mid E_{z}^{c s}\right) \operatorname{det}\left(\tilde{\pi}_{f^{-n}(z)} \mid E_{f^{-n}(z)}^{c s}\right)^{-1},
$$

and since $\tilde{\pi} \circ \pi=\tilde{\pi}$,

$$
\operatorname{det}\left(\pi_{z} \mid T_{z} \Omega\right)=\operatorname{det}\left(\tilde{\pi}_{z} \mid T_{z} \Omega\right) \operatorname{det}\left(\tilde{\pi}_{z} \mid E_{z}^{c s}\right)^{-1}
$$

Therefore for $y \in \Omega^{\prime}$,

$$
\frac{\operatorname{det}\left(f^{-n} \mid E_{y}^{c s}\right) \operatorname{det}\left(\pi \mid T_{y} \Omega^{\prime}\right)}{\operatorname{det}\left(f^{-n} \mid E_{h(y)}^{c s}\right) \operatorname{det}\left(\pi \mid T_{h(y)} \Omega^{\prime \prime}\right)}=\frac{\operatorname{det}\left(\tilde{f}^{-n} \mid \tilde{F}_{y}\right) \operatorname{det}\left(\tilde{\pi} \mid T_{y} \Omega^{\prime}\right)}{\operatorname{det}\left(\tilde{f}^{-n} \mid \tilde{F}_{h(y)}\right) \operatorname{det}\left(\tilde{\pi} \mid T_{h(y)} \Omega^{\prime \prime}\right)} \cdot \frac{\operatorname{det}\left(\tilde{\pi} \mid E_{f^{-n}(h(y))}^{c s}\right)}{\operatorname{det}\left(\tilde{\pi} \mid E_{f^{-n}(y)}^{c s}\right)}
$$

and then (6.3) follows from the fact that $h(y) \in W^{u}(y)$, hence $\operatorname{dist}\left(f^{-n}(h(y)), f^{-n}(y)\right)$ converges uniformly to zero as $n \rightarrow \infty$. 
We introduce the coordinate system $\chi$ as follows. Consider an analytic system of coordinates around $x_{0}, \rho: \bar{U} \times \bar{V} \subset \mathbb{R}^{\operatorname{dim} E^{u}} \times \mathbb{R}^{\operatorname{dim} E^{c s}} \rightarrow X$, such that $x_{0}=\rho(0,0)$ and $\rho_{t}:=\rho(t, \cdot): \bar{V} \rightarrow X$ are transversals to $W^{u}$ for each $t \in \bar{U}$. Denote the image of $\rho(t, \cdot)$ by $\Omega_{t} \subset X$. For a point $x$ close to $x_{0}$ define its coordinates $\chi^{-1}(x)=(t, v) \in \bar{U} \times \bar{V}$ by the condition that $x=\Omega_{t} \cap W_{0, \text { loc }}^{u}(\rho(0, v))$.

That is, $\chi(t, v)=h_{t}(\rho(0, v))$, where $h_{t}: \Omega_{0} \rightarrow \Omega_{t}$ is the holonomy map along $W^{u}$. The domain of $\chi$ will be an open subset $U \times V \subset \bar{U} \times \bar{V}$ containing the origin. $\chi$ has the required analyticity properties. It remains to show that its Jacobian is as desired.

Note that $\rho^{-1} \circ \chi(t, v)=\left(t, \tilde{h}_{t}(v)\right)$, where $\tilde{h}_{t}=\rho_{t}^{-1} \circ h_{t} \circ \rho_{0}$. Hence, up to some $C^{\omega}$ functions and changes of variable, the Jacobian of $\chi$ is given by $J(t, y):=J_{\Omega_{t}}^{\Omega_{0}}(y)$, with $t \in U$ and $y \in \Omega_{0}$. We are done once we prove the following.

Claim 2. $J(t, y)$ is analytic in $t \in U$, uniformly with respect to $y \in \Omega_{0}$.

Proof. Choose as $\tilde{F}$ an analytic bundle on $X$ which is complementary to $E^{u}$, and denote $\mathcal{D}(z):=\operatorname{det}\left(\tilde{f} \mid \tilde{F}_{z}\right)$. In view of (6.2), one can rewrite (6.3) as

$$
J(t, y)=\lim _{n \rightarrow \infty} \frac{\operatorname{det}\left(\tilde{f}^{-n} \mid \tilde{F}_{y}\right)}{\prod_{k=0}^{n-1} \mathcal{D} \circ f^{-k}\left(h_{t}(y)\right)} \cdot \frac{\operatorname{det}\left(\tilde{\pi} \mid T_{y} \Omega_{0}\right)}{\operatorname{det}\left(\tilde{\pi} \mid T_{h_{t}(y)} \Omega_{t}\right)} .
$$

Note that $\mathcal{D} \in C_{W^{u}}^{\omega}(X, \mathbb{R})$. Indeed, one can choose locally trivializing frames of $T X$ consisting of the union of a frame for $E^{u}$ and an analytic frame for $\tilde{F}$. The vector fields in the former can be chosen to be in $C_{W^{u}}^{\omega}$. Then all the entries of the matrix of $D f$ with respect to these frame are in $C_{W^{u}}^{\omega}(X, \mathbb{R})$, hence the same holds for $\tilde{f}$ as well. We also obtain that $\tilde{\pi}: T X \rightarrow \tilde{F}$ has entries in $C_{W^{u}}^{\omega}(X, \mathbb{R})$.

Given a product of positive real valued functions $P=a_{n-1} \cdots a_{1} a_{0}$, its derivative is given by $D P=P\left[D\left(\log a_{n-1}\right)+\cdots+D\left(\log a_{1}\right)+D\left(\log a_{0}\right)\right]$.

Denote by $D_{u}$ the differential in the $U$-variable (i.e. along $W^{u}$ ), and let

$$
J_{n}(t, y):=\frac{\operatorname{det}\left(\tilde{f}^{-n} \mid \tilde{F}_{y}\right)}{\prod_{k=0}^{n-1} \mathcal{D} \circ f^{-k}\left(h_{t}(y)\right)} \cdot \frac{\operatorname{det}\left(\tilde{\pi} \mid T_{y} \Omega_{0}\right)}{\operatorname{det}\left(\tilde{\pi} \mid T_{h_{t}(y)} \Omega_{t}\right)} .
$$

Then

$$
\begin{aligned}
D_{u} J_{n}(t, y)= & -J_{n}(t, y)\left(D_{u} \log \left(\operatorname{det}\left(\tilde{\pi} \mid T_{h_{t}(y)} \Omega_{t}\right)\right)+\sum_{k=0}^{n-1} D_{u} \log \left(\mathcal{D} \circ f^{-k}\left(h_{t}(y)\right)\right)\right) \\
= & -J_{n}(t, v)\left(D_{u} \log \left(\operatorname{det}\left(\tilde{\pi} \mid T_{h_{t}(y)} \Omega_{t}\right)\right)\right. \\
& \left.+\left.\left.\left.\sum_{k=0}^{n-1} D_{u} \log \mathcal{D}\right|_{f^{-k}\left(h_{t}(y)\right)} D_{u} f^{-k}\right|_{h_{t}(y)} D_{u} h_{t}\right|_{y}\right) .
\end{aligned}
$$

Due to the factor $D_{u} f^{-k}$ and the uniform convergence of $J_{n}$ one obtains that $D_{u} J_{n}$ converges uniformly, hence $J$ is differentiable along the leaf and

$$
D_{u} \log (J(t, y))=-\left(D_{u} \log \left(\operatorname{det}\left(\tilde{\pi} \mid T_{h_{t}(y)} \Omega_{t}\right)\right)+\sum_{k=0}^{\infty} D_{u} \log \left(\mathcal{D} \circ f^{-k}\left(h_{t}(y)\right)\right)\right) .
$$

We proved so far that the Jacobian of $W^{u}$ is $C^{1}$. To obtain that it is $C^{\omega}$, notice that the right-hand side in (6.5) is analytic, uniformly with respect to $y$. Indeed, each factor 
involved in the sum in (6.4) is analytic in $t$, uniformly with respect to $y$, and the sum remains convergent when evaluated on the complexification of the unstable leaves.

We can now complete the proof of Theorem 2.2.

Proof of Theorem 2.2 for $K=\omega$. The discussion of $\S 5$ shows that under the hypotheses of the theorem, $\mathbf{P}(x, y):=\left(x, P_{x}(y)\right)$ maps stable/unstable leaves into stable/unstable leaves. Since these foliations are analytic, one obtains that $\mathbf{P}$ is uniformly $C^{\omega}$ along their leaves. The center foliation can be dealt with as in $\S 5$. Therefore $\mathbf{P}$ has the desired smoothness along three (four if $\mathcal{A}=\mathbb{R}$ ) $C^{\omega}$ foliations which span $M \times N$. Their Jacobians satisfy the requirements of Theorem 6.1, either by the hypotheses or Theorem 6.4.

Acknowledgements. We are indebted to the referee for the proof of Theorem 2.4. It simplifies and extends our original proof of the case $G \subset \mathrm{GL}(d, \mathbb{R})$. Our approach was based on the construction of stable and unstable foliations for certain skew-products. Since this construction has applications beyond Theorem 2.4 (see $[\mathbf{B N}]$ and [KNT]), we present it in the Appendix.

We would like to thank A. Katok for pointing out to us that de la Llave's counterexample is relevant to the problem we consider.

Appendix. Existence of stable and unstable foliations

We describe here how one can obtain an analogue of the stable and unstable foliations for some skew-products. Using these, one can prove Theorem 2.4 for $G \subset \operatorname{GL}(d, \mathbb{R})$ in a similar way to Theorem 2.3: if two cocycles $\beta$ and $\tilde{\beta}$ are cohomologous, i.e. $\beta(n, x)=P\left(T^{n} x\right) \tilde{\beta}(n, x) P(T x)^{-1}$, and $P$ is Hölder of a prescribed order, then the corresponding foliations are mapped one onto the other.

Given a cocycle $\beta: \mathcal{A} \times M \rightarrow \mathrm{GL}(d, \mathbb{R})$ over the action $T: \mathcal{A} \times M \rightarrow M$, one can consider $f \in \operatorname{Diff}^{\infty}\left(M \times \mathbb{R}^{d}\right)$ defined by $f(x, \xi)=\left(T^{1} x, \beta(1, x) \xi\right), x \in M, \xi \in \mathbb{R}^{d}$.

Note that although we speak about invariant foliations for $f$, the statement is about a family of functions with values in the group.

By $W_{0}^{s}, W_{0}^{c}$ and $W_{0}^{u}$ we denote the stable, center and unstable foliations of $T^{1}$.

THEOREM A.1. Let $K, T$ and $\lambda_{ \pm}$be as in Theorem 2.4, and $\beta$ a $C^{K}$-cocycle taking values in $\mathrm{GL}(d, \mathbb{R})$. Consider

$$
\begin{gathered}
\tilde{\mu}_{+}=\lim _{n \rightarrow \infty} \sup _{x \in M}\|\beta(n, x)\|^{1 / n}, \\
\tilde{\mu}_{-}=\lim _{n \rightarrow \infty} \inf _{x \in M}\left\|\beta(n, x)^{-1}\right\|^{-1 / n},
\end{gathered}
$$

where $\|\cdot\|$ denotes the matrix norm.

Assume that $\lambda_{-}<\tilde{\mu}_{-} \cdot \tilde{\mu}_{+}^{-1}$. Then there are $C^{K}$-functions $\gamma_{x}: W_{0}^{s}(x) \rightarrow \operatorname{GL}(d, \mathbb{R})$, $x \in M$, satisfying the conditions:

(1) $\gamma_{x}(x)=I$;

(2) the family of 'graphs' $W^{s}(x, \xi):=\left\{\left(t, \gamma_{x}(t) \xi\right): t \in W_{0}^{s}(x)\right\}, x \in M, \xi \in \mathbb{R}^{d}$, gives an $f$-invariant foliation of $M \times \mathbb{R}^{d}$. 
These functions are defined by the formula

$$
\gamma_{x}(t)=\lim _{n \rightarrow \infty} \beta(n, t)^{-1} \beta(n, x), \quad t \in W_{0}^{s}(x),
$$

and depend continuously on the point $x \in M$ in the $C^{K}$-topology.

The graph $\operatorname{Graph}\left(\gamma_{x}\right) \subset W_{0}^{s}(x) \times \mathrm{GL}(d, \mathbb{R})$ is characterized by

$$
\begin{aligned}
(t, u) \in \operatorname{Graph}\left(\gamma_{x}\right) & \Leftrightarrow \lim _{n \rightarrow \infty}\left\|\beta(n, x)^{-1}\right\| \cdot\|\beta(n, x)-\beta(n, t) u\|=0 \\
& \Leftrightarrow \lim _{n \rightarrow \infty} v_{-}^{-n}\|\beta(n, x)-\beta(n, t) u\|=0
\end{aligned}
$$

for $\lambda_{-} \cdot \tilde{\mu}_{+}<\nu_{-}<\tilde{\mu}_{-}$. In particular, the family $\left\{\gamma_{x}\right\}_{x \in M}$ is the unique family of functions which satisfies conditions (1) and (2) above and is uniformly Lipschitz on $W_{0,10 c}^{s}$.

Similar results hold for the unstable direction.

Proof. Note that the invariance property of the family $\left\{W^{s}(x, \xi)\right\}_{x \in M, \xi \in \mathbb{R}^{d}}$ amounts to the relation

$$
\beta(1, t) \gamma_{x}(t)=\gamma_{T^{1} x}\left(T^{1} t\right) \beta(1, x), \quad t \in W_{0}^{s}(x),
$$

that is

$$
\gamma_{x}(t)=\beta(1, t)^{-1} \gamma_{T^{1} x}\left(T^{1} t\right) \beta(1, x) .
$$

Iterating (A.1) we obtain that

$$
\gamma_{x}(t)=\beta(n, t)^{-1} \cdot \gamma_{T^{n} x}\left(T^{n} t\right) \cdot \beta(n, x) .
$$

This suggests that we define $\gamma_{x}$ by the formula used in the theorem.

Consider the functions $\gamma_{x, n}: W_{0}^{s}(x) \rightarrow \operatorname{GL}(n, \mathbb{R})$ given by $\gamma_{x, n}(t):=\beta(n, t)^{-1}$. $\beta(n, x)$. Denote $\beta(1, \cdot)$ by $\beta(\cdot)$.

Given $\lambda_{-} \cdot \tilde{\mu}_{+}<v_{-}<\tilde{\mu}_{-}$, choose $\kappa_{-}>\lambda_{-}$and $\nu_{+}>\tilde{\mu}_{+}$such that $v_{+} \cdot v_{-}^{-1} \cdot \kappa_{-}<1$. There is a constant $C_{0}>0$ such that for $n \geq 0$ and $t \in W_{0, \text { loc }}^{s}(x)$,

$$
\begin{gathered}
\left\|\left.D T^{n}\right|_{E^{s}}\right\| \leq C_{0} \kappa_{-}^{n}, \\
\operatorname{dist}_{M}\left(T^{n} t, T^{n} x\right) \leq C_{0} \kappa_{-}^{n} \operatorname{dist}_{M}(t, x), \\
\sup _{y \in M}\left\|\beta(n, y)^{-1}\right\| \leq C_{0} \nu_{-}^{-n}, \\
\sup _{y \in M}\|\beta(n, y)\| \leq C_{0} v_{+}^{n} .
\end{gathered}
$$

Note that it is enough to construct each $\gamma_{x}$ on $W_{0, \text { loc }}^{s}(x)$ only, and then extend them by (A.1).

CLAIM 1. The limit $\gamma_{x}:=\lim _{n \rightarrow \infty} \gamma_{x, n}$ exists uniformly on $W_{0, \mathrm{loc}}^{s}(x)$ and defines a continuous function $\gamma_{x}: W_{0, \mathrm{loc}}^{s}(x) \rightarrow \mathrm{GL}(d, \mathbb{R})$. The functions $\gamma_{x}$ defined this way satisfy (A.1). Moreover, $\gamma_{x}(t)$ is bounded by a constant which is independent of $x \in M$ and $t \in W_{0, \mathrm{loc}}^{s}(x)$. Denote this constant by $C_{*}$.

Since $\gamma_{x}(t)=\gamma_{x^{\prime}}(t) \gamma_{x}\left(x^{\prime}\right)$ for $t, x^{\prime} \in W_{0, \text { loc }}^{s}(x)$, the family $\left\{W^{s}(x, \xi): x \in M, \xi \in \mathbb{R}^{d}\right\}$ gives a partition of $M \times \mathbb{R}^{d}$. 
Proof. We show that the sequence $\left\{\gamma_{x, n}\right\}$ is uniformly Cauchy. Let $m>n$ be positive integers and let $t \in W_{0, \text { loc }}^{s}(x)$. Then:

$$
\begin{aligned}
\left\|\gamma_{x, m}(t)-\gamma_{x, n}(t)\right\| \leq & \sum_{k=n}^{m-1}\left\|\gamma_{x, k+1}(t)-\gamma_{x, k}(t)\right\| \\
= & \sum_{k=n}^{m-1} \| \beta(k, t)^{-1} \beta\left(T^{k+1} t\right)^{-1} \beta\left(T^{k+1} x\right) \beta(k, x) \\
& -\beta(k, t)^{-1} \beta\left(T^{k+1} t\right)^{-1} \beta\left(T^{k+1} t\right) \beta(k, x) \| \\
\leq & \sum_{k=n}^{m-1} C_{0}^{2} \cdot v_{-}^{-k-1} \cdot v_{+}^{k} \cdot\left\|\beta\left(T^{k+1} x\right)-\beta\left(T^{k+1} t\right)\right\| \\
\leq & C_{0}^{3} v_{+}^{-1} \sum_{k=n}^{m-1}\left(\nu_{-}^{-1} \cdot v_{+} \cdot \kappa_{-}\right)^{k+1}\|\beta\|_{\text {Lip }} \operatorname{dist}_{M}(t, x) \\
\leq & C\left(v_{-}^{-1} \cdot v_{+} \cdot \kappa_{-}\right)^{n},
\end{aligned}
$$

where the constant $C$ does not depend on $m, n, x$ or $t$.

The rest of the Claim follows from the identities $\gamma_{x, n}(t)=\gamma_{x^{\prime}, n}(t) \gamma_{x, n}\left(x^{\prime}\right)$ and $\gamma_{x, n+1}(t)=\beta(1, t)^{-1} \gamma_{T^{1} x, n}\left(T^{1} t\right) \beta(1, x)$.

CLAIM 2. The functions $\gamma_{x}: W_{0, \mathrm{loc}}^{s}(x) \rightarrow \mathrm{GL}(d, \mathbb{R})$ are Lipschitz and their Lipschitz norm is bounded by some constant $L_{*}$, independently of $x \in M$.

Proof. Let $t, t^{\prime} \in W_{0, \mathrm{loc}}^{s}(x)$ and $n>0$. Then:

$$
\begin{aligned}
&\left\|\gamma_{x, n+1}(t)-\gamma_{x, n+1}\left(t^{\prime}\right)\right\| \\
&= \| \beta^{-1}(t) \cdots \beta^{-1}\left(T^{n} t\right) \beta^{-1}\left(T^{n} x\right) \cdots \beta^{-1}(x) \\
&-\beta^{-1}\left(t^{\prime}\right) \cdots \beta^{-1}\left(T^{n} t^{\prime}\right) \beta^{-1}\left(T^{n} x\right) \cdots \beta^{-1}(x) \| \\
& \leq \sum_{k=0}^{n} \| \beta^{-1}\left(t^{\prime}\right) \cdots \beta^{-1}\left(T^{k-1} t^{\prime}\right) \beta^{-1}\left(T^{k} t\right) \beta^{-1}\left(T^{k+1} t\right) \cdots \beta^{-1}\left(T^{n} t\right) \beta\left(T^{n} x\right) \cdots \beta(x) \\
&-\beta^{-1}\left(t^{\prime}\right) \cdots \beta^{-1}\left(T^{k} t^{\prime}\right) \beta^{-1}\left(T^{k+1} t\right) \cdots \beta^{-1}\left(T^{n} t\right) \beta\left(T^{n} x\right) \cdots \beta(x) \| \\
& \leq \sum_{k=0}^{n}\left\|\beta\left(k, t^{\prime}\right)^{-1}\right\| \cdot\left\|\beta^{-1}\left(T^{k} t^{\prime}\right)-\beta^{-1}\left(T^{k} t\right)\right\| \cdot\left\|\gamma_{T^{k+1} x, n-k}\left(T^{k+1} t\right)\right\| \cdot\|\beta(k+1, x)\| \\
& \leq \sum_{k=0}^{n}\left(C_{0} v_{-}^{-k}\right) \cdot\left(\left\|\beta^{-1}\right\|_{\text {Lip }} C_{0} \kappa_{-}^{k} \operatorname{dist}_{M}\left(t^{\prime}, t\right)\right) \cdot C_{*} \cdot\left(C_{0} v_{+}^{k+1}\right) \\
& \leq L_{*} \operatorname{dist}_{M}\left(t, t^{\prime}\right),
\end{aligned}
$$

where $L_{*}$ does not depend on $n, x, t$ or $t^{\prime}$. Now take the limit as $n \rightarrow \infty$.

ClaIM 3. The functions $\gamma_{x}: W_{0, \text { loc }}^{s}(x) \rightarrow \mathrm{GL}(d, \mathbb{R})$ are $C^{1}$.

Proof. We denote by $D_{s}$ the derivative along $W_{0}^{s}$. Then for $t \in W_{0, \mathrm{loc}}^{s}(x)$,

$$
\left.D_{s} \gamma_{x, n+1}\right|_{t}=D_{s}\left[\beta^{-1}(t) \beta^{-1}\left(T^{1} t\right) \cdots \beta^{-1}\left(T^{n} t\right) \cdot \beta\left(T^{n} x\right) \cdots \beta(x)\right]
$$




$$
\begin{aligned}
= & \left.\sum_{k=0}^{n} \beta^{-1}(t) \cdots \beta^{-1}\left(T^{k-1} t\right) \cdot D_{s} \beta^{-1}\right|_{T^{k} t} \cdot \beta^{-1}\left(T^{k+1} t\right) \cdots \beta^{-1}\left(T^{n} t\right) \\
& \left.\cdot \beta\left(T^{n} x\right) \cdots \beta(x) D_{s} T^{k}\right|_{t} \\
= & \left.\left.\sum_{k=0}^{n} \beta(k, t)^{-1} \cdot D_{s} \beta^{-1}\right|_{T^{k} t} \cdot \beta\left(n-k, T^{k+1} t\right)^{-1} \cdot \beta(n+1, x) \cdot D_{s} T^{k}\right|_{t} .
\end{aligned}
$$

We now use (A.2) and the cocycle relation to obtain

$$
\begin{aligned}
\left.D_{s} \gamma_{x, n+1}\right|_{t}= & \left.\sum_{k=0}^{n} \beta(k, t)^{-1} \cdot D_{s} \beta^{-1}\right|_{T^{k} t} \cdot \gamma_{T^{k+1} x}\left(T^{k+1} t\right) \\
& \cdot \beta\left(n-k, T^{k+1} x\right)^{-1} \gamma_{T^{n+1} x}^{-1}\left(T^{n+1} t\right) \beta\left(n-k, T^{k+1} x\right) \\
& \left.\cdot \beta(k+1, x) D_{s} T^{k}\right|_{t} \\
= & \left.\left.\sum_{k=0}^{n} \beta(k, t)^{-1} \cdot D_{s} \beta^{-1}\right|_{T^{k} t} \cdot \gamma_{T^{k+1} x}\left(T^{k+1} t\right) \cdot \beta(k+1, x) D_{s} T^{k}\right|_{t} \\
& +\left.\sum_{k=0}^{n} \beta(k, t)^{-1} D_{s} \beta^{-1}\right|_{T^{k} t} \cdot \gamma_{T^{k+1} x}\left(T^{k+1} t\right) \\
& \left.\cdot \Omega\left(n-k, T^{k+1} x, T^{k+1} t\right) \cdot \beta(k+1, x) D_{s} T^{k}\right|_{t},
\end{aligned}
$$

where

$$
\Omega(k, y, s)=\beta(k, y)^{-1} \cdot\left[\gamma_{T^{k} y}\left(T^{k} s\right)-I\right] \cdot \beta(k, y) .
$$

Since $\gamma_{x}$ is Lipschitz with norm $L_{*}$, we see that for $t \in W_{0, \text { loc }}^{s}(x)$ and $k \leq n$,

$$
\left\|\Omega\left(n-k, T^{k+1} x, T^{k+1} t\right)\right\| \leq L_{*} C_{0}^{3} v_{-}^{-(n-k)} \cdot v_{+}^{n-k} \cdot \kappa_{-}^{n+1} \leq L_{*} C_{0}^{3} .
$$

Using this estimate in (A.4), and once more (A.2), we obtain that $\left.D_{s} \gamma_{x, n}\right|_{t}$ converges uniformly to

$$
\left.\left.\sum_{k=0}^{\infty} \gamma_{x}(t) \cdot \beta(k, x)^{-1} \cdot \gamma_{T^{k} x}\left(T^{k} t\right)^{-1} D_{s} \beta^{-1}\right|_{T^{k} t} \cdot \gamma_{T^{k+1} x}\left(T^{k+1} t\right) \cdot \beta(k+1, x) D_{s} T^{k}\right|_{t} .
$$

Note that this is an absolutely convergent series, which is continuous with respect to $x \in M$ and $t \in W_{0}^{s}(x)$.

CLAIM 4. The functions $\gamma_{x}: W_{0}^{s}(x) \rightarrow \mathrm{GL}(d, \mathbb{R})$ are $C^{K}$ and depend continuously on the point $x \in M$ in the $C^{K}$-topology.

Proof. From the comment preceding Lemma 2.3 in [LMM] it follows that

$$
\left\|D_{s}^{l} T^{k}\right\| \leq(l+1) ! C_{l} k^{l+1} \kappa_{-}^{k-l+1} .
$$

Then a direct computation from the expression (A.5) of $D_{s} \gamma_{x}$ shows that the higher derivatives of $\gamma_{x}$ can be found by term-wise differentiation of (A.5). The resulting series also have the required continuity. This solves the case $K \leq \infty$.

The case $K=\omega$ follows from the observation that the uniform convergence proved in Claim 1 also holds on the complexification of $W_{0, \text { loc }}^{s}\left(W_{0}^{s}\right.$ is a $C^{\omega}$-foliation by Theorem 3.1(a)). 
Indeed, since $M, T^{1}$ and $\beta(1, \cdot)$ are analytic, there is a complex analytic manifold $\tilde{M}$ containing $M$ and analytic extensions of $T^{1}$ and $\beta(1, \cdot)$ to $\tilde{T}: \tilde{M}_{0} \rightarrow \tilde{M}$ and respectively $\tilde{\beta}_{0}: \tilde{M}_{0} \rightarrow \operatorname{GL}(d, \mathbb{C})$, where $\tilde{M}_{0}$ is an open neighborhood of $M$ in $\tilde{M}$. Each leaf $W_{0, \text { loc }}^{s}(x), x \in M$, has a uniform extension to a complex analytic sub-manifold $\tilde{W}_{0, \text { loc }}^{s}(x)$ of $\tilde{M}_{0}, \tilde{T}\left(\tilde{W}_{0, \text { loc }}^{s}(x)\right) \subset \tilde{W}_{0, \text { loc }}^{s}\left(T^{1}(x)\right)$, and $\tilde{T}$ acts as a contraction on these complexified leaves. On $\tilde{M}^{s}:=\bigcup_{x \in M} \tilde{W}_{0, \text { loc }}^{s}(x)$ one can define a 'cocycle' $\tilde{\beta}_{+}: \mathbb{Z}_{+} \times \tilde{M}^{s} \rightarrow \operatorname{GL}(d, \mathbb{C})$ over $\tilde{T}$. The corresponding constants $\tilde{\lambda}_{-}$and $\tilde{\mu}_{ \pm}$can be made as close to $\lambda_{-}$, respectively $\tilde{\mu}_{ \pm}$, as desired by shrinking $\tilde{M}_{0}$ (note that the limits defining these numbers are actually infima).

Then $\tilde{\gamma}_{x, n}(t):=\tilde{\beta}_{+}(n, t)^{-1} \cdot \tilde{\beta}_{+}(n, x)$ is defined on $\tilde{W}_{0, \mathrm{loc}}^{s}(x)$ for $x \in M$ and is an analytic extension of $\gamma_{x, n}$. The computation of Claim 1 carries over to $\tilde{\gamma}_{x, n}$ and we obtain the existence of a uniform limit as $n \rightarrow \infty$, bounded on $\tilde{W}_{0, \text { loc }}^{s}(x)$ by some constant which is independent of $x$. We conclude that $\gamma_{x}$ is analytic and depends continuously in $C^{\omega}$ on $x \in M$.

Note that for the unstable foliation we have to use a different extension of the cocycle, but the constants are still given by $\tilde{\mu}_{ \pm}$.

Claim 5. Given $(t, u) \in W_{0}^{s}(x) \times \operatorname{GL}(d, \mathbb{R})$, one has

$$
\begin{aligned}
(t, u) \in \operatorname{Graph}\left(\gamma_{x}\right) & \Rightarrow \lim _{n \rightarrow \infty} v_{-}^{-n}\|\beta(n, x)-\beta(n, t) u\|=0 \\
& \Rightarrow \lim _{n \rightarrow \infty}\left\|\beta(n, x)^{-1}\right\| \cdot\|\beta(n, x)-\beta(n, t) u\|=0 \\
& \Rightarrow(t, u) \in \operatorname{Graph}\left(\gamma_{x}\right) .
\end{aligned}
$$

Since the first implication follows from the conclusion of Claim 2 and conditions (1) and (2), this proves the characterization as uniformly Lipschitz functions as well.

Proof. Indeed, for $n$ large enough so that $T^{n} t \in W_{0, \mathrm{loc}}^{s}\left(T^{n} x\right)$, one obtains by (A.2), condition (1), Claim 2 and (A.3) that

$$
\begin{aligned}
v_{-}^{-n}\left\|\beta(n, x)-\beta(n, t) \gamma_{x}(t)\right\| & =v_{-}^{-n}\left\|\left(I-\gamma_{T^{n} x}\left(T^{n} t\right)\right) \beta(n, x)\right\| \\
& \leq L_{*} \operatorname{dist}_{M}\left(T^{n} t, T^{n} x\right) v_{-}^{-n}\|\beta(n, x)\| \\
& \leq C\left(\lambda_{-} \cdot v_{-}^{-1} \cdot v_{+}\right)^{n},
\end{aligned}
$$

which proves the first implication. The second follows by (A.3).

For the third, write $u=\gamma_{x}(t) v, v \in \mathrm{GL}(d, \mathbb{R})$. Then, using the first implication and the inequality $\|A B\| \geq\left\|A^{-1}\right\|^{-1}\|B\|$ for $A, B \in \operatorname{Mat}(d, \mathbb{R}), A$ invertible, one obtains

$$
\begin{aligned}
& \left\|\beta(n, x)^{-1}\right\| \cdot\|\beta(n, x)-\beta(n, t) u\| \\
& \quad=\left\|\beta(n, x)^{-1}\right\| \cdot\left\|\beta(n, x)(I-v)+\left(I-\gamma_{T^{n} x}\left(T^{n} t\right)\right) \beta(n, x) v\right\| \\
& \quad \geq\|I-v\|-C\left(\lambda_{-} \cdot v_{-}^{-1} \cdot v_{+}\right)^{n}\|v\|,
\end{aligned}
$$

which yields the desired conclusion. 


\section{REFERENCES}

[A] D. V. Anosov. Geodesic flows on closed Riemannian manifolds with negative curvature. Proc. Stek. Inst. 90 (1967), 1-235.

[BN] H. Bercovici and V. Niţică. A Banach algebra version of the Livsic theorem. Discrete and Continuous Dynamical Systems, 4(3) (1998), 523-534.

[B] A. Borel. Compact Clifford-Klein forms of symmetric spaces. Topology 2 (1963), 111-122.

[BP] M. I. Brin and Ya. B. Pesin. Partially hyperbolic dynamical systems. Math. USSR Izvestia 8 (1974), 177-218.

[CEG] P. Collet, H. Epstein and G. Gallavotti. Perturbations of geodesic flows on surfaces of constant negative curvature and their mixing properties. Commun. Math. Phys. 95 (1984), 61-112.

[GK1] V. Guillemin and D. Kazhdan. On the cohomology of certain dynamical systems. Topology 19 (1980), 291-299.

[GK2] V. Guillemin and D. Kazhdan. Some inverse spectral results for negatively curved 2-manifolds. Topology 19 (1980), 301-313.

[HPS] M. Hirsch, C. Pugh and M. Shub. Invariant Manifolds (Lecture Notes in Mathematics, 583). Springer, 1977.

[H] S. Hurder. Rigidity of Anosov actions of higher rank lattices. Ann. Math. 135 (1992), 361-410.

[HuK] S. Hurder and A. Katok. Differentiability, rigidity and Godbillon-Vey classes for Anosov flows. Publ. Math. Inst. Hautes Etudes Sci. 72 (1990), 5-61.

[J] J.-L. Journé. A regularity lemma for functions of several variables. Revista Matematica Iberoamericana 4(2) (1988), 187-193.

[KL] A. Katok and J. Lewis. Local rigidity for certain groups of toral automorphisms. Israel J. Math. 75(2-3) (1992), 203-242.

[KNT] A. Katok, V. Niţică and A. Török. Nonabelian cohomology of abelian Anosov actions. Ergod. Th. \& Dynam. Sys. to appear.

[L1] A. Livsic. Homology properties of $U$ systems. Math. Notes. 10 (1971), 758-763.

[L2] A. Livsic. Cohomology of dynamical systems. Math. USSR, Izvestia 6 (1972), 1278-1301.

[Lla1] R. de la Llave. Analytic regularity of solutions of Livsic's cohomology equations and some applications to analytic conjugacy of hyperbolic dynamical systems. Ergod. Th. \& Dynam. Sys. 17 (1997), 649-662.

[Lla2] R. de la Llave. Smooth conjugacy and S-B-R measures for uniformly and non-uniformly hyperbolic systems. Commun. Math. Phys. 150 (1992), 289-320.

[LMM] R. de la Llave, J. M. Marco and R. Moriyon. Canonical perturbation theory of Anosov systems and regularity results for Livsic cohomology equation. Ann. Math. 123 (1986), 537-612.

[M] J. N. Mather. Characterization of Anosov diffeomorphisms. Indag. Math. 30 (1968), 479-483.

[NT1] V. Niţică and A. Török. Cohomology of dynamical systems and rigidity of partially hyperbolic actions of higher rank lattices. Duke Math. J. 79 (1995), 751-810.

[NT2] V. Niţică and A. Török. Regularity results for the solution of Livsic cohomology equation with values in diffeomorphism groups. Ergod. Th. \& Dynam. Sys. 16 (1996), 325-333.

[P] Ya. B. Pesin. On the existence of invariant fibering for the diffeomorphisms of a smooth manifold. Math. USSR Sbornik 20(2) (1973), 213-222.

[PS] C. Pugh and M. Shub. Ergodicity of Anosov actions. Invent. Math. 15 (1972), 1-23. 